\title{
Basic partitions and combinations of group actions on the circle: A new approach to a theorem of Kathryn Mann
}

\author{
Shigenori Matsumoto
}

\begin{abstract}
Let $\Pi_{g}$ be the surface group of genus $g(g \geq 2)$, and denote by $\mathcal{R}_{\Pi_{g}}$ the space of the homomorphisms from $\Pi_{g}$ into the group of the orientation preserving homeomorphisms of $S^{1}$. Let $2 g-2=k l$ for some positive integers $k$ and $l$. Then the subset of $\mathcal{R}_{\Pi_{g}}$ formed by those $\varphi$ which are semiconjugate to $k$-fold lifts of some homomorphisms and which have Euler number $e u(\varphi)=l$ is shown to be clopen. This leads to a new proof of the main result of Kathryn Mann 4 from a completely different approach.
\end{abstract}

\section{Introduction}

Let $S^{1}=\mathbb{R} / \mathbb{Z}$ and denote the canonical projection by $\pi: \mathbb{R} \rightarrow S^{1}$. Denote by $T: \mathbb{R} \rightarrow \mathbb{R}$ the translation by one: $T(x)=x+1$.

Notations 1.1. Let $\mathcal{H}=$ Homeo $_{+}\left(S^{1}\right)$ denote the group of the orientation preserving homeomorphisms of $S^{1}$, and for any group $G, \mathcal{R}_{G}=\operatorname{Homo}(G, \mathcal{H})$ the set of the homomorphisms from $G$ to $\mathcal{H}$.

Definition 1.2. A map $h: S^{1} \rightarrow S^{1}$ is called degree one monotone if there is a nondecreasing (not necessarily continuous) map $\tilde{h}: \mathbb{R} \rightarrow \mathbb{R}$ such that $\tilde{h} \circ T=T \circ \tilde{h}$ and $\pi \circ \tilde{h}=h \circ \pi$.

Denote

$$
\mathcal{R}_{G}^{*}=\left\{\varphi \in \mathcal{R}_{G} \mid \exists x \in S^{1} \text { such that } \varphi(g)(x)=x, \forall g \in G\right\} .
$$

Definition 1.3. Two homomorphisms $\varphi^{1}, \varphi^{2} \in \mathcal{R}_{G}$ are called semiconjugate, denoted $\varphi^{1} \sim \varphi^{2}$, if either $\varphi^{1}, \varphi^{2} \in \mathcal{R}_{G}^{*}$ or $\varphi^{1}, \varphi^{2} \in \mathcal{R}_{G} \backslash \mathcal{R}_{G}^{*}$ and there is a degree one monotone map $h: S^{1} \rightarrow S^{1}$ such that $\varphi^{2}(g) \circ h=h \circ \varphi^{1}(g)$ for any $g \in G$.

The proof of the following proposition can be found in Appendix A.

Proposition 1.4. The semiconjugacy is an equivalence relation.

Definition 1.5. Let $F^{i} \subset S^{1}$ be a $\varphi^{i}(G)$-invariant subset $\left(\varphi^{i} \in \mathcal{R}_{G}, i=1,2\right)$. A map $\xi: F^{1} \rightarrow F^{2}$ is called $\left(\varphi^{1}, \varphi^{2}\right)$-equivariant if $\xi \circ \varphi^{1}(g)=\varphi^{2}(g) \circ \xi$ on $F^{1}$ for any $g \in G$.

1991 Mathematics Subject Classification. 37E10.

Key words and phrases. basic partition, combination, surface group, Euler number.

The author is partially supported by Grant-in-Aid for Scientific Research (C) No. 25400096. 
We have the following easy proposition.

Proposition 1.6. Let $F^{i} \subset S^{1}$ be a $\varphi^{i}(G)$-invariant subset $\left(\varphi^{i} \in \mathcal{R}_{G}, i=\right.$ $1,2)$, and assume there is a cyclic order preserving $\left(\varphi^{1}, \varphi^{2}\right)$-equivariant bijection $\xi: F^{1} \rightarrow F^{2}$. Then we have $\varphi^{1} \sim \varphi^{2}$.

Proof. Two homomorphisms $\varphi_{1} \in \mathcal{R}_{G}^{*}$ and $\varphi_{2} \in \mathcal{R}_{G} \backslash \mathcal{R}_{G}^{*}$ can never satisfy the condition of the proposition. So one may assume $\varphi^{i} \in \mathcal{R}_{G} \backslash \mathcal{R}_{G}^{*}$. There is an order preserving bijection $\widetilde{\xi}: \pi^{-1}\left(F^{1}\right) \rightarrow \pi^{-1}\left(F^{2}\right)$ such that $\widetilde{\xi} \circ T=T \circ \widetilde{\xi}$ and $\xi \circ \pi=\pi \circ \widetilde{\xi}$. Define $\widetilde{h}: \mathbb{R} \rightarrow \mathbb{R}$ by

$$
\widetilde{h}(\widetilde{x})=\inf \left\{\widetilde{\xi}(\widetilde{y}) \mid \widetilde{y} \in[\widetilde{x}, \infty) \cap \pi^{-1}\left(F^{1}\right)\right\} .
$$

Then $\widetilde{h} \circ T=T \circ \widetilde{h}$, and there is a monotone degree one map $h: S^{1} \rightarrow S^{1}$ such that $h \circ \pi=\pi \circ \widetilde{h}$. Now $\left(\varphi^{1}, \varphi^{2}\right)$-equivariance of $\xi$ implies that $h \circ \varphi^{1}(g)=\varphi^{2}(g) \circ h$ $(\forall g \in G)$.

Definition 1.7. A homomorphism $\varphi \in \mathcal{R}_{G}$ is called type 0 if there is a $\varphi(G)$ invariant probability measure on $S^{1}$.

If there is a finite $\varphi(G)$-orbit or if the action of $\varphi(G)$ is free, then $\varphi$ is type 0 . If $\varphi$ is type 0 and $\varphi \sim \varphi^{\prime}$, then $\varphi^{\prime}$ is also type 0 . If $\varphi$ is not type 0 , then the minimal set of $\varphi$ is unique, either a Cantor set or the whole $S^{1}$. In the latter case we say that $\varphi$ is minimal.

Definition 1.8. For $\varphi$ not of type 0, a minimal homomorphism which is semiconjugate to $\varphi$ is denoted by $\varphi_{\sharp}$, and called a minimal model.

A minimal model $\varphi_{\sharp}$ always exists and is unique up to topological conjugacy for $\varphi$ not of type 0 . For any $k \geq 2$, let $\pi_{k}: S^{1} \rightarrow S^{1}$ be the $k$-fold covering map, that is, $\pi_{k}(x+\mathbb{Z})=k x+\mathbb{Z}$.

Definition 1.9. For $k \in \mathbb{N}, \psi \in \mathcal{R}_{G}$ is called a $k$-fold lift of $\varphi \in \mathcal{R}_{G}$ if for any $g \in G$, it holds that $\varphi(g) \circ \pi_{k}=\pi_{k} \circ \psi(g)$.

Definition 1.10. For $k \in \mathbb{N}$, a homomorphism $\varphi \in \mathcal{R}_{G}$ is called type $k$ if it satisfies the following conditions.

(1) $\varphi$ is not type 0 .

(2) A minimal model $\varphi_{\sharp}$ is a $k$-fold lift of some homomorphism in $\mathcal{R}_{G}$.

(3) $k$ is the maximal among those which satisfy (2).

For $k \geq 0$, the set of type $k$ homomorphisms is denoted by $\mathcal{R}_{G}(k)$.

Thus type 1 homomorphisms are those homomorphisms which are not type 0 and whose minimal model cannot be a $k$-fold lift for any $k \geq 2$.

The group $\mathcal{H}$ is a topological group with the uniform convergence topology, defined by the metric:

$$
d(f, h)=\sup _{x \in S^{1}}|f(x)-h(x)| \text { for } f, h \in \mathcal{H} .
$$

The space $\mathcal{R}_{G}$ is equipped with the following topology. Given $\varphi \in \mathcal{R}_{G}, g \in G$ and $\varepsilon>0$, let

$$
U(\varphi ; g, \varepsilon)=\left\{\varphi^{\prime} \in \mathcal{R}_{G} \mid d\left(\varphi^{\prime}(g), \varphi(g)\right)<\varepsilon\right\}
$$


The topology with subbase $U(\varphi ; g, \varepsilon)$ is called the weak topology. When the group $G$ is finitely generated, this coincides with the usual topology of uniform convergence on generators. The following proposition will be proven in the next section.

Proposition 1.11. For any group $G$ and $k \geq 1$, the subset $\mathcal{R}_{G}(0)$ is closed and $\bigcup_{1 \leq i \leq k} \mathcal{R}_{G}(i)$ is open in $\mathcal{R}_{G}$.

This is best possible, for example for free groups. However for groups of a special kind, one can expect that some component of $\mathcal{R}_{G}(k), k \geq 2$, is also open. The purpose of this paper is to consider this problem for the surface group $\Pi_{g}$, $g \geq 2$. The group $\Pi_{g}$ is the fundamental group of the closed oriented surface of genus $g$, and has a presentation:

$$
\Pi_{g}=\left\langle A_{1}, B_{1}, \ldots, A_{g}, B_{g} \mid\left[A_{1}, B_{1}\right] \cdots\left[A_{g}, B_{g}\right]=e\right\rangle .
$$

Given $\varphi \in \mathcal{R}_{\Pi_{g}}$, its Euler number eu $(\varphi) \in \mathbb{Z}$ is defined by

$$
\left.\left.\widetilde{\varphi\left(A_{1}\right)}, \widetilde{\varphi\left(B_{1}\right)}\right] \cdots \widetilde{\varphi\left(A_{g}\right)}, \widetilde{\varphi\left(B_{g}\right)}\right]=T^{e u(\varphi)},
$$

where for $f \in \mathcal{H}, \widetilde{f}$ denotes an arbitrary lift of $f$ to a homeomorphism of $\mathbb{R}$. The map eu: $\mathcal{R}_{\Pi_{g}} \rightarrow \mathbb{Z}$ is continuous, and thus $e u^{-1}(i)$ is clopen in $\mathcal{R}_{\Pi_{g}}$ for any $i \in \mathbb{Z}$. We have the following classical theorem [8, [9, called the Milnor-Wood inequality.

ThEOREM 1.12. The inverse image eu $u^{-1}(i)$ is nonempty if and only if $|i| \leq$ $2 g-2$.

For homomorphisms with the extremal values of Euler number, we have the following result $\mathbf{7}$. (In fact, the pathwise connectedness below is not mentioned in that paper. But it is an easy consequence of the main theorem.)

THEOREM 1.13. The inverse image $E_{+}=e u^{-1}(2 g-2)$ is pathwise connected, and if $\varphi, \varphi^{\prime} \in E_{+}$, then $\varphi \sim \varphi^{\prime}$. The same thing holds true for $E_{-}=e u^{-1}(-2 g+2)$.

Assume $e u(\varphi)=2 g-2$ and $2 g-2=k l$ for some positive integers $k, l$. Choose an arbitary $k$-fold lift $\widehat{\varphi\left(A_{j}\right)}\left(\operatorname{resp} . \widehat{\varphi\left(B_{j}\right)}\right)$ of $\varphi\left(A_{j}\right)\left(\right.$ resp. $\left.\varphi\left(B_{j}\right)\right)$ for $j=1, \ldots, g$. Then we have

$$
\left[\widehat{\varphi\left(A_{1}\right)}, \widehat{\varphi\left(B_{1}\right)}\right] \ldots\left[\widehat{\varphi\left(A_{g}\right)}, \widehat{\varphi\left(B_{g}\right)}\right]=\mathrm{Id} \text {. }
$$

In fact, this is obtained by taking a quotient by the action of $T^{l}$ of the formula:

$$
\left.\left.\widetilde{\left[\varphi\left(A_{1}\right)\right.}, \widetilde{\varphi\left(B_{1}\right)}\right] \cdots \widetilde{\left[\left(A_{g}\right)\right.}, \widetilde{\varphi\left(B_{g}\right)}\right]=T^{2 g-2}=T^{k l} \text {. }
$$

Thus we have a $k$-fold lift of $\varphi$ once we choose $k$-fold lifts of the generators arbitrarily. We shall denote the $k$-fold lifts of $\varphi$ by $\psi_{j}, 1 \leq j \leq k^{2 g}$. The following result is immediate.

Proposition 1.14. We have eu $\left(\psi_{j}\right)=l$.

The main result of the present paper is the following.

TheOREM 1.15. Assume $2 g-2=k l$ for some positive integers $k$ and $l$. Then the subset $\mathrm{eu}^{-1}(l) \cap \mathcal{R}_{\Pi_{g}}(k)$ is clopen in $\mathcal{R}_{\Pi_{g}}$.

The closedness of $e u^{-1}(l) \cap \mathcal{R}_{\Pi_{g}}(k)$ follows from Proposition 1.11, In fact, we have

$$
e u^{-1}(l) \cap \mathcal{R}_{\Pi_{g}}(k)=e u^{-1}(l) \backslash \cup_{1 \leq j \leq k-1} \mathcal{R}_{\Pi_{g}}(j),
$$

where $e u^{-1}(l)$ is closed and $\cup_{1 \leq j \leq k-1} \mathcal{R}_{\Pi_{g}}(j)$ is open.

For the openness, we use the following concept. 
Definition 1.16. For any group $G$, a homomorpism $\varphi \in \mathcal{R}_{G}$ is said to be locally stable if any homomorphism $\varphi^{\prime} \in \mathcal{R}_{G}$ sufficiently near to $\varphi$ is semi-conjugate to $\varphi$.

The openness follows from the following theorem.

TheOREM 1.17. Any homomorphism of $\mathrm{eu}^{-1}(l) \cap \mathcal{R}_{\Pi_{g}}(k)$ is locally stable.

Let $Z_{j}$ be the connected component of $\mathcal{R}_{\Pi_{g}}$ which contains the above lift $\psi_{j}$, $1 \leq j \leq k^{2 g}$. Then we have the following corollary.

Corollary 1.18. Any two homomorphisms of the same component $Z_{j}$ are mutually semi-conjugate.

The same result has been obtained by K. Mann [4, based upon extensive use of algorythms in 2. This paper contains a completely different approach. Also there is a quite simple proof for diffeomorphisms due to J. Bowden 1 .

We shall prove Proposition 1.11 in Section 2, and Theorem 1.17 in Sections $4-7$. We give an outline of Sections 4 and 5 in Section 3. It seems that our method provides a new and elementary proof of the main result of $\mathbf{7}$, but we do not pursue it in the present paper. Throughout the paper, we use the following notations.

Notations 1.19. • The positive cyclic order of $S^{1}$ is denoted by $\prec$.

- Given two distinct points $a, b \in S^{1},[a, b]=\left\{x \in S^{1}, a \prec x \prec b\right\}$.

For a subset $X$ of $S^{1}$, we denote

- $C \sqsubset X$ if $C$ is a connected component of $X$,

- $X_{\sharp}$ the union of the closures of the connected components of $S^{1} \backslash X$,

- $X_{*}=X \cap X_{\sharp}$.

We abbreviate

- BP for "basic partition", BC for "basic configuration" and COP for "cyclic order preserving".

ACKNOWLEDGEMEnt. Hearty thanks are due to the referee, whose valuable comments are helpful for the improvement of the paper.

\section{Proximal actions}

In this section, $G$ is to be an arbitrary group, countable or not. This section is devoted to the proof of Proposition 1.11. Let us begin by showing that $\mathcal{R}_{G}(0)$ is a closed subset of $\mathcal{R}_{G}$. Let $\varphi$ be any homomorphism from the closure of $\mathcal{R}_{G}(0)$. Let us denote by $\mathcal{P}\left(S^{1}\right)$ the space of the probability measures on $S^{1}$, equipped with the weak* topology. In order to show $\varphi$ admits an invariant probability measure, it is sufficient to prove that for any finite subset $\left\{g_{i}\right\} \subset G$, there is a probability measure invariant by $\varphi\left(g_{i}\right)_{*}: \mathcal{P}\left(S^{1}\right) \rightarrow \mathcal{P}\left(S^{1}\right)$, thanks to the finite intersection property of the compact set $\mathcal{P}\left(S^{1}\right)$. Choose

$$
\varphi_{n} \in \bigcap_{i} U\left(\varphi ; g_{i}, 1 / n\right) \cap \mathcal{R}_{G}(0),
$$

where $U(\cdot)$ is introduced in (1.1), and let $\mu_{n} \in \mathcal{P}\left(S^{1}\right)$ be a $\varphi_{n}(G)$-invariant measure. Since the maps $\varphi_{n}\left(g_{i}\right)_{*}$ and $\varphi\left(g_{i}\right)_{*}$ are continuous and $\varphi_{n}\left(g_{i}\right)_{*}$ converges to $\varphi\left(g_{i}\right)_{*}$ pointwise, an accumulation point of $\left\{\mu_{n}\right\}$ is the desired measure. 
Now let us turn to show that $\mathcal{R}_{G}(1)$ is an open subset of $\mathcal{R}_{G}$. The argument is based upon the following Theorem 2.2 due to É. Ghys (p.362, [3] ), whose proof is included in Appendix B. To state it, we make a definition.

Definition 2.1. A homomorphism $\varphi \in \mathcal{R}_{G}$ is called proximal if for any closed interval $I \subset S^{1}, \inf _{g \in G}|\varphi(g) I|=0$, where $|\cdot|$ denotes the diameter.

Theorem 2.2. For any $\varphi \in \mathcal{R}_{G}, \varphi \in \mathcal{R}_{G}(1)$ if and only if a minimal model $\varphi_{\sharp}$ is proximal.

Definition 2.3. Given $x, y \in S^{1}$, a sequence $\left\{f_{n}\right\} \subset \mathcal{H}$ is called an $(x, y)$ sequence if for any $\varepsilon>0$, there is $N$ such that if $n \geq N, f_{n}$ maps the complement of the $\varepsilon$-neighbourhood of $x$ into the $\varepsilon$-neighbourhood of $y$.

Lemma 2.4. For any $x, y \in S^{1}$ and $\varphi \in \mathcal{R}_{G}(1)$, there is an $(x, y)$-sequence in $\varphi_{\sharp}(G)$.

Proof. For any $x \in S^{1}$, define

$$
E_{x}=\left\{y \in S^{1} \mid \exists(x, y) \text {-sequence in } \varphi_{\sharp}(G)\right\} .
$$

By Theorem 2.2. $E_{x}$ is nonempty for any $x \in S^{1}$. On the other hand, it is easy to show that $E_{x}$ is closed and $\varphi_{\sharp}(G)$-invariant. Therefore we have $E_{x}=S^{1}$.

There is a bounded 2-cocycle $c$ of the group $\mathcal{H}$ defined by

$$
c(f, h)=\tau(\widetilde{f} \circ \widetilde{h})-\tau(\widetilde{f})-\tau(\widetilde{h}),
$$

where $\tilde{f}$ (resp. $\tilde{h}$ ) is an arbitrary lift of $f($ resp. $h$ ) to $\mathbb{R}$, and $\tau(\cdot)$ stands for the translation number. As is well known, its $L^{\infty}$ norm satisfies $\|c\|=1$. For $\varphi \in \mathcal{R}_{G}$, the pull back cocycle $\varphi^{*} c$ lies in the second bounded cocycle group $Z_{b}^{2}(G)$ of $G$ and satisfies $\left\|\varphi^{*} c\right\| \leq 1$. It is known [6] that $\varphi^{*} c=0$ if and only if $\varphi \in \mathcal{R}_{G}(0)$. For other $\mathcal{R}_{G}(k)$, we have the following.

Lemma 2.5. For any $\varphi \in \mathcal{R}_{G}$ and $k \geq 1, \varphi \in \mathcal{R}_{G}(k)$ if and only if $\left\|\varphi^{*} c\right\|=1 / k$.

Proof. It suffices to show only the following implication:

$$
\varphi \in \mathcal{R}_{G}(k) \Rightarrow\left\|\varphi^{*} c\right\|=1 / k, \quad \forall k \geq 1,
$$

since the opposite implication follows from this. First of all, let us show (2.1) for $k=1$. Let $\varphi_{\sharp}$ be a minimal model of any $\varphi \in \mathcal{R}_{G}(1)$. Choose four points $x \prec y \prec z \prec u \prec x$ in $S^{1}$. By Lemma 2.4, there are a $(y, x)$-sequence $f_{n}$ and an $(u, z)$-sequence $h_{n}$ in $\varphi_{\sharp}(G)$. Let $\widetilde{f}_{n}$ and $\widetilde{h}_{n}$ be the lifts of $f_{n}$ and $h_{n}$ such that $\tau\left(\widetilde{f}_{n}\right)=\tau\left(\widetilde{h}_{n}\right)=0$. One can choose lifts of the four points so that $\widetilde{x}<\widetilde{y}<\widetilde{z}<\widetilde{u}<$ $T(\widetilde{x})$. See Figure 1 for this and the next argument.

For $n$ large, $\widetilde{h}_{n}$ admits a fixed point, say $\tilde{u}_{n}$, near $\tilde{u}$. Now consider the composite $\widetilde{f}_{n} \circ \widetilde{h}_{n}$. Clearly we have $\widetilde{u}_{n}<\widetilde{f}_{n} \circ \widetilde{h}_{n}\left(\widetilde{u}_{n}\right)<T\left(\widetilde{u}_{n}\right)$. On the other hand, if we choose $\widetilde{u}^{\prime}$ very near to $\tilde{u}$ so that $\widetilde{u}^{\prime}>\widetilde{u}$. Then for any large $n$, we have $\widetilde{f}_{n} \circ \widetilde{h}_{n}\left(u^{\prime}\right)>T\left(u^{\prime}\right)$. (See Figure 1.) This shows $\tau\left(\tilde{f}_{n} \circ \widetilde{h}_{n}\right)=1$. Therefore $c\left(f_{n}, h_{n}\right)=1$ and $\left\|\varphi^{*} c\right\|=$ $\left\|\varphi_{\sharp}^{*} c\right\|=1$, as is required. Also it is not difficult to show that the above inequalities also show the following.

For any $\varphi^{\prime} \in \mathcal{R}_{G}$ sufficiently near to $\varphi \in \mathcal{R}_{G}(1)$, we have $\left\|\left(\varphi^{\prime}\right)^{*} c\right\|=1$.

To show (2.1) for $k \geq 1$, choose any $\varphi \in \mathcal{R}_{G}(k)$, with $\varphi_{\sharp}$ a $k$-fold lift of some $\psi \in \mathcal{R}_{G}$. Clearly $\psi \in \mathcal{R}_{G}(1)$. Moreover the cocycle $\varphi^{*} c=\varphi_{\sharp}^{*} c$ is precisely $(1 / k) \psi^{*} c$. This shows $\left\|\varphi^{*} c\right\|=1 / k$. 

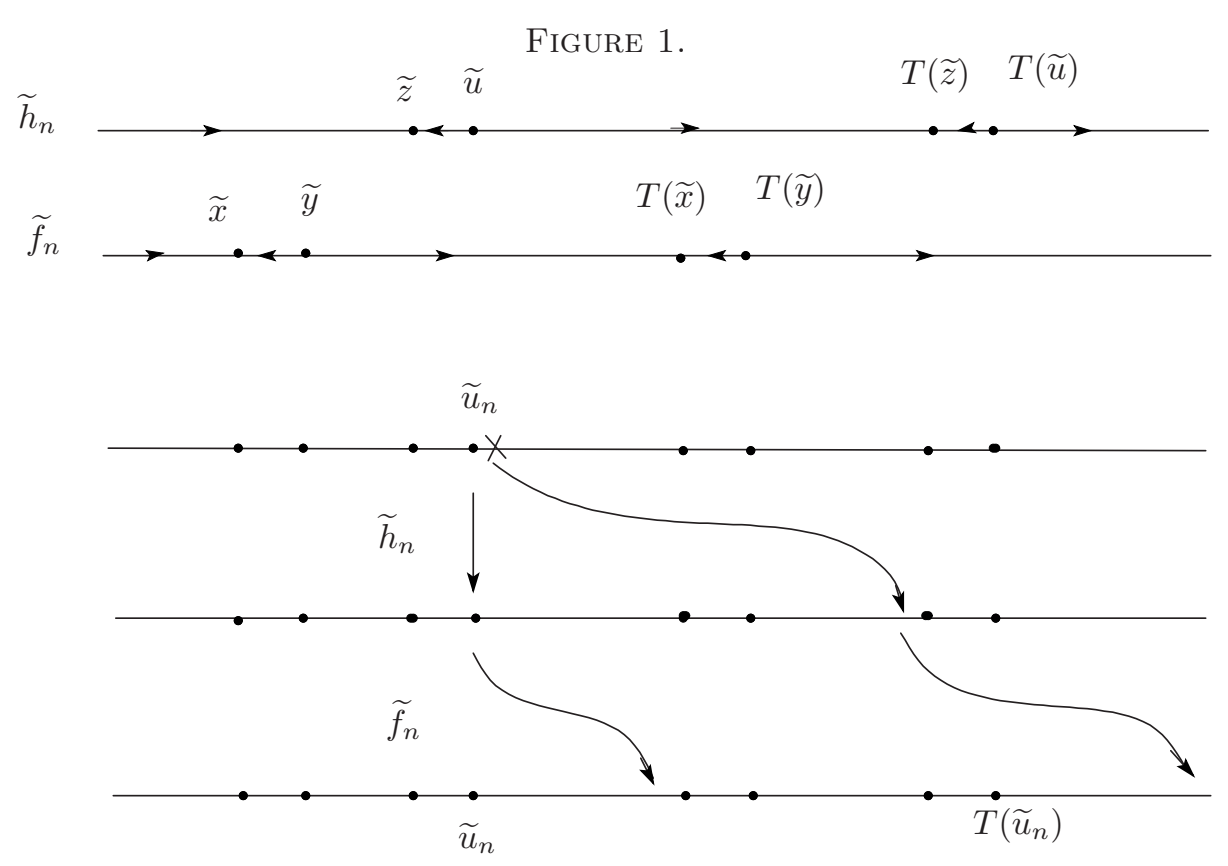

Now the openness of $\mathcal{R}_{G}(1)$ follows from Lemma 2.5 and (2.2). The proof that the set $\bigcup_{1 \leq i \leq k} \mathcal{R}_{G}(i)$ is open is left to the reader.

\section{Outline}

Before getting into a detailed proof of Theorem 1.17 we shall give an outline of its first two steps. The basic idea is that a homomorphism in $e u^{-1}(l) \cap \mathcal{R}_{\Pi_{g}}(k)$ of Theorem 1.17 has the following very special property: There is a finite set, say $R$, of $S^{1}$ such that the knowledge about how the generators of the group moves points of $R$ completely determines the semiconjugacy class of the homomorphism.

First of all, let us explain this phenomenon in a much simpler example. Let $\Gamma$ be the free group on two generators $A$ and $B$. Let $\varphi \in \mathcal{R}_{\Gamma}$ and denote $a=\varphi(A)$ and $b=\varphi(B)$. Assume that $\tau([\widetilde{a}, \widetilde{b}])=1$, where $\widetilde{a}$ (resp. $\widetilde{b}$ ) is an arbitrary lift of $a$ (resp. $b$ ). Then one can show that such $\varphi$ belongs to a single semiconjugacy class. This will be actually done in Section 4. But we can present a rough outline here.

By the assumption $\tau([\widetilde{a}, \widetilde{b}])=1$, there is a fixed point $x \in S^{1}$ of $[a, b]$ such that

$$
x \prec b^{-1}(x) \prec a^{-1} b^{-1}(x) \prec b a^{-1} b^{-1}(x) \prec[a, b](x)=x .
$$

See Figure 2 left.

The homeomorphism $a$ maps the long interval $\left[b a^{-1} b^{-1}(x), a^{-1} b^{-1}(x)\right]$ onto a subinterval $\left[x, b^{-1}(x)\right]$. Therefore there is a fixed point of $a$ in the open interval $\left(x, b^{-1}(x)\right)$. There is also a fixed point in $\left(a^{-1} b^{-1}(x), b a^{-1} b^{-1}(x)\right)$. Likewise $b$ admits at least two fixed points, one in $\left(b^{-1}(x), a^{-1} b^{-1}(x)\right)$, another in $\left(b a^{-1} b^{-1}(x), x\right)$.

Let $R$ be the set of four points in Figure 2 left, and set $S=\left\{A, A^{-1}, B, B^{-1}\right\}$. Let $R^{2}=\bigcup_{s \in S} \varphi(s) R$. Then $R^{2}$ contains $R$, and has 8 more points. The configuration of $R^{2}$ in $S^{1}$ is determined uniquely. Likewise if we set $R^{3}=\bigcup_{s \in S} \varphi(s) R^{2}$, 


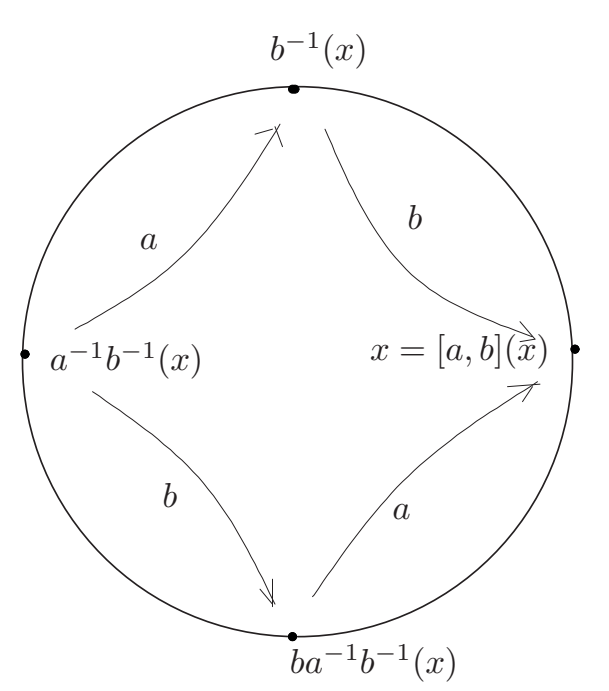

FIGURE $2 . \quad y=a b(y)$

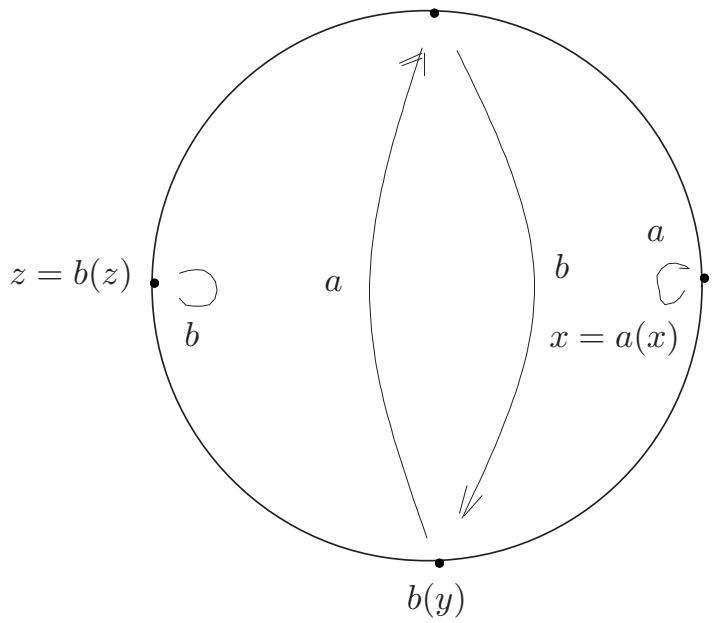

FiguRE 3.

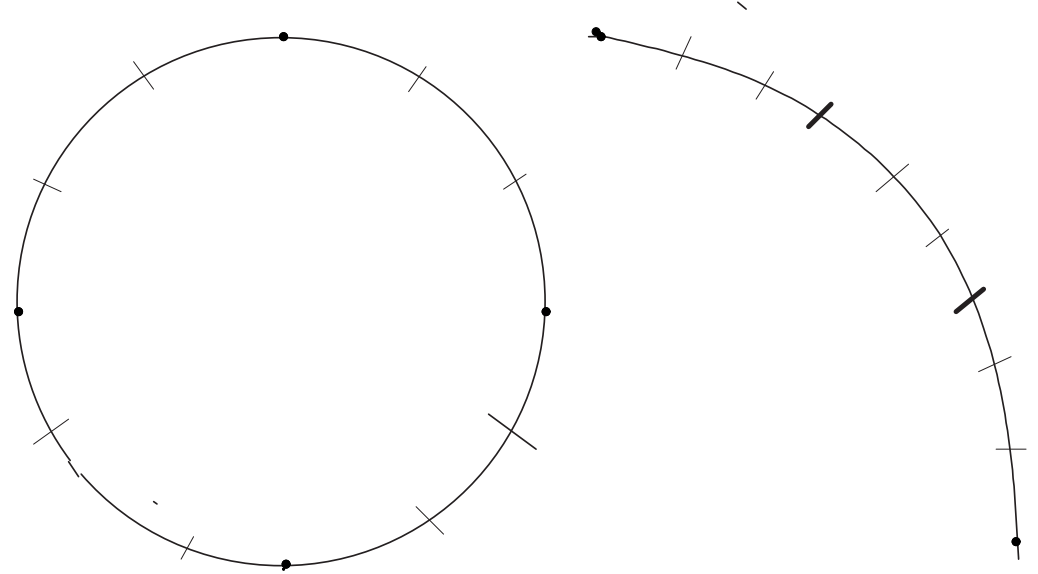

then its configuration is also unique. See Figure 3. The left depicts $R^{2}$ and the right a part of $R^{3}$. This way, we can determine the configuration of the whole orbit $\varphi(\Gamma) x$, which, according to Proposition [1.6 implies that the semiconjugacy class of $\varphi$ is uniquely determined. The actual proof can be organized as an induction.

Here is another example of this kind. See Figure 2 right. This is also a homomorphism $\varphi$ from the free group on two generators $A$ and $B$, and we denote $a=\varphi(A)$ and $b=\varphi(B)$. The homeomorphism $a$ (resp. $b$ ) has a fixed point $x$ (resp. $z)$, and we have $y=a b(y)$ for the point $y$ in the figure. Clearly $c(a, b)=1$ and any homomorphism with $c(a, b)=1$ has a configuration as in Figure 2 right. Again one can show that such $\varphi$ belongs to a single semiconjugacy class. That is, if we let $R$ be the set of four points $x, y, z$ and $b(y)$, then the same thing holds with this $R$.

What is good about these partitions $R$ is the following. Let $\psi$ be any $k$-fold lift of $\varphi$. Then the pull back image $\pi_{k}^{-1}(R)$ has the same property: it determines the semiconjugacy class of the homomorphism $\psi$. 
Figure 4.
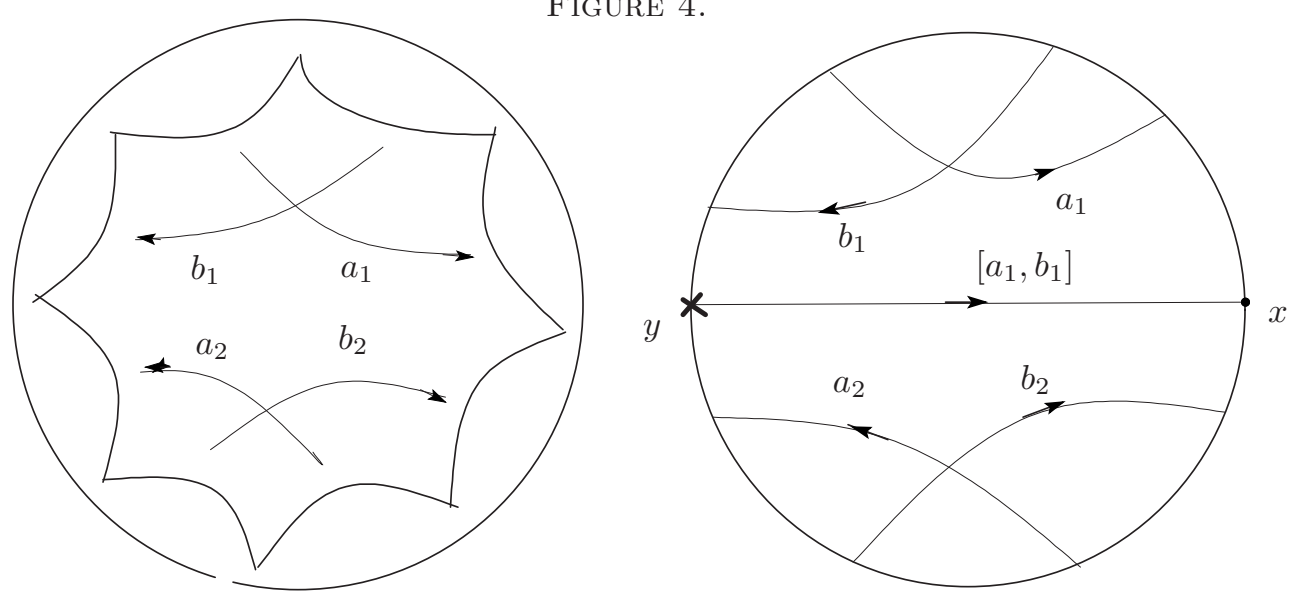

What is not good is that this kind of partitions are difficult to find out. To show Theorem 1.17 we need something more.

Let us consider a Fuchsian representation $\varphi \in \mathcal{R}_{\Pi_{2}}$ of the surface group $\Pi_{2}$ of genus 2 such that $e u(\varphi)=2$. One can assume the elements $a_{\nu}=\varphi\left(A_{\nu}\right)$ and $b_{\nu}=\varphi\left(B_{\nu}\right)(\nu=1,2)$ are the hyperbolic motions in Figure 4 left. The axes of $a_{\nu}$, $b_{\nu}$ and $\left[a_{1}, b_{1}\right]=\left[b_{2}, a_{2}\right]$ are depicted in Figure 4 right. Let $x$ and $y$ be the fixed points of $\left[a_{1}, b_{1}\right]$. See Figure 5 for parts of orbits of $x$ and $y$.

The set $R$ of fourteen points there is enough to determine the semiconjugacy class of the homomorphism $\varphi$. In fact, the configuration of $R$ immediately implies that $\operatorname{eu}(\varphi)=2$, and by [7, the semiconjugacy class is unique. However when we consider a 2 -fold lift $\psi$ of $\varphi$, it is not clear if the inverse image $\pi_{2}^{-1}(R)$ actually determines the semiconjugacy class or not. To cope with the problem, we need an algorythm to determine the orbits of $x$ and $y$, which can be inherited to a $k$-fold cover. But this is not according to the word length of the elements of $\Pi_{2}$.

Consider the amalgamated product

$$
\Pi_{2}=\Gamma_{1} *_{\Lambda} \Gamma_{2},
$$

where $\Gamma_{\nu}$ is the subgroup generated by $A_{\nu}$ and $B_{\nu}$ and $\Lambda$ generated by $\left[A_{1}, B_{1}\right]=$ $\left[B_{2}, A_{2}\right]$. First we consider the homomorphism $\varphi_{\nu}=\left.\varphi\right|_{\Gamma_{\nu}}$. This is a homomorphism from the free group $\Gamma_{\nu}$ on two generators such that $\tau\left(\left[\widetilde{a}_{\nu}, \widetilde{b}_{\nu}\right]\right)=1$, and the previous observation works. However notice that one can define the set $R$ of four points in Figure 2 in two different ways: one from the orbit of $x$, the other $y$. It is more natural and more convenient to consider disjoint four intervals (instead of points). For $\Gamma_{1}$, they are $E_{1}=[y, x]$ and its iterates in Figure 6 . The complement of the four intervals is denoted by $P_{1}$. The stabilizer (in $\Gamma_{1}$ ) of $E_{1}$ is $\Lambda$, and the limit set of the Fuchsian group $\Gamma_{1}$ is contained in $P_{1}$. Likewise in the right figure, the four intervals are $E_{2}=[x, y]$ and its iterates. The complement is denoted by $P_{2}$.

For $\gamma_{\nu} \in \Gamma_{\nu}$, the configuration of the orbits $\varphi_{\nu}\left(\gamma_{\nu}\right)(x)$ and $\varphi_{\nu}\left(\gamma_{\nu}\right)(y)$ is determined just by the data in Figure 6 inductively on the word length of $\gamma_{\nu}$, as we have explained. They are contained in $P_{\nu}$. The actual proof is given in Section 4 , where we call such subsets $P_{\nu}$ basic partitions. The complementary intervals $E_{\nu}$ is called 
Figure 5.

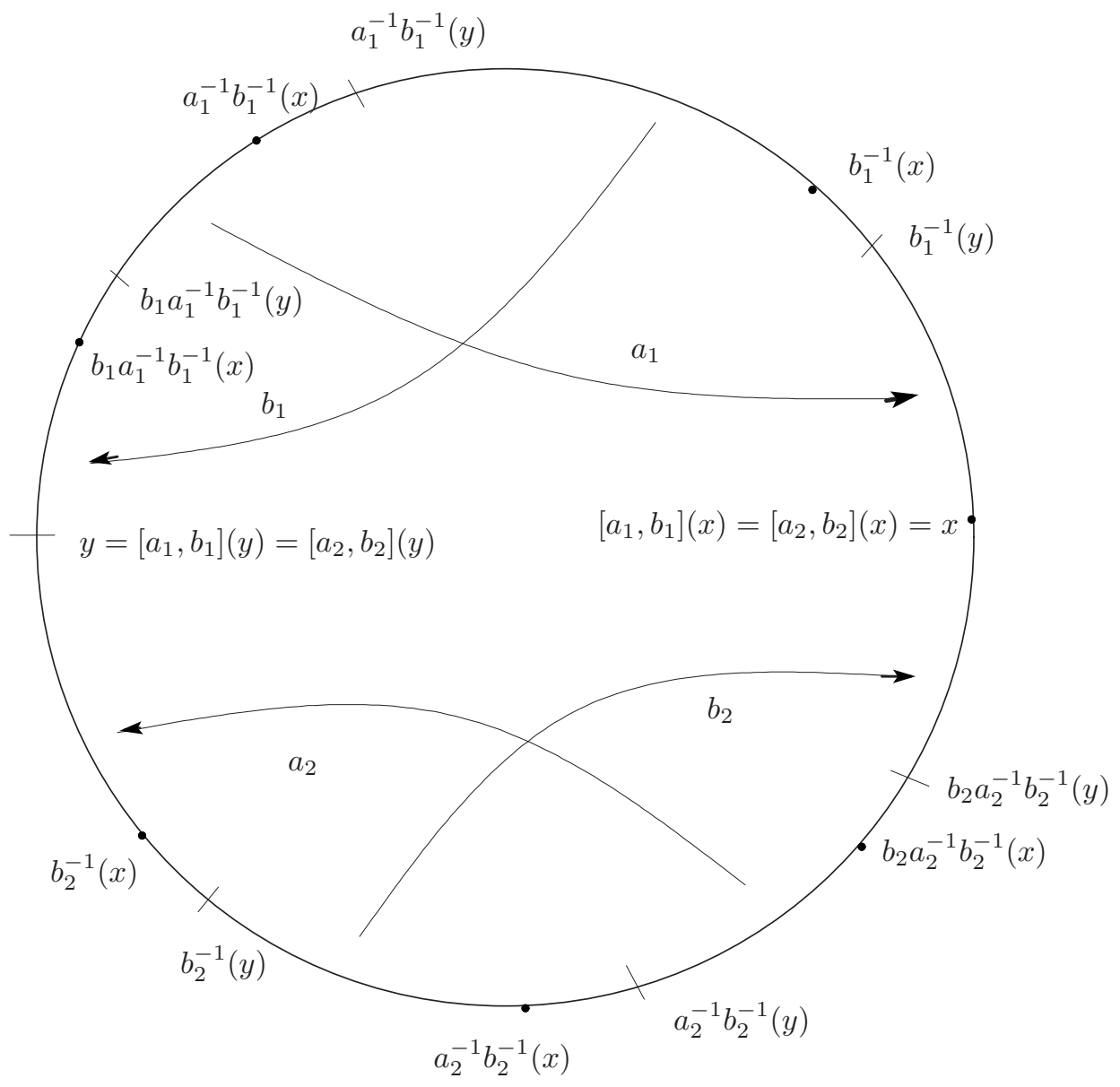

Figure 6.
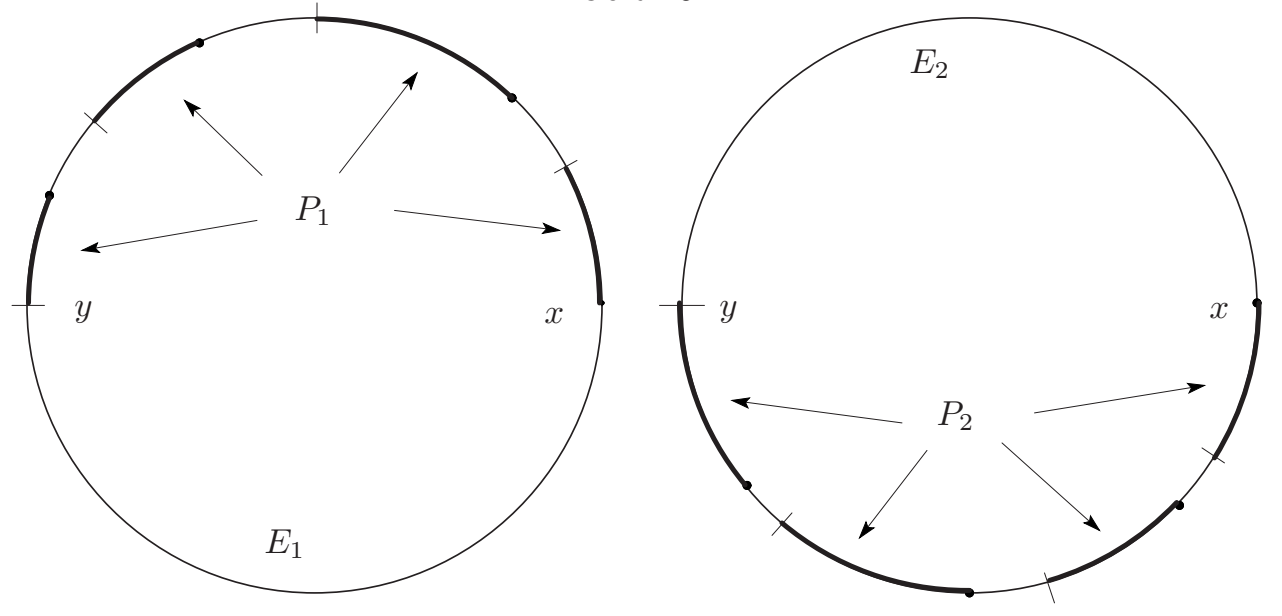
FiguRE 7.

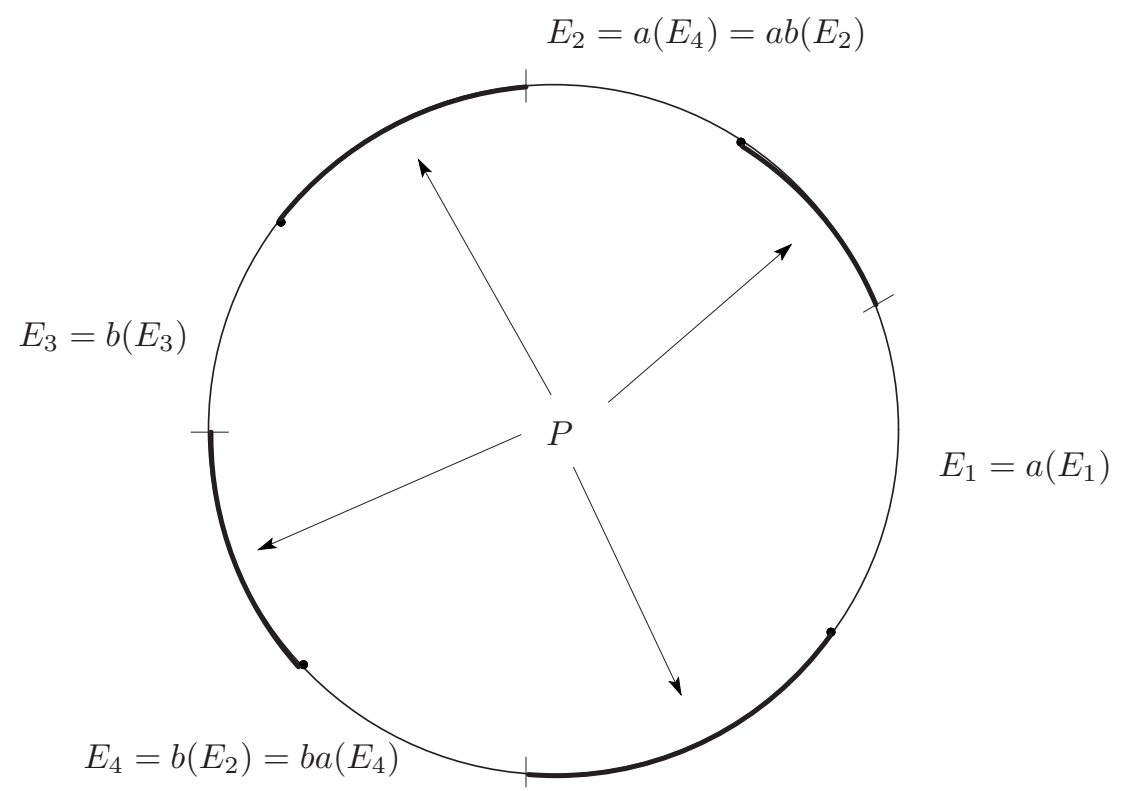

the entrance of $\Lambda$ to $P_{\nu}$. As we explained, the stabilizer of $E_{\nu}$ (in $\Gamma_{\nu}$ ) is $\Lambda$. The entrances $E_{1}$ and $E_{2}$ satisfy the conditions; $E_{1} \cup E_{2}=S^{1}$ and Int $E_{1} \cap \operatorname{Int} E_{2}=\emptyset$. They are said to be combinable. Now the whole orbits $\varphi(g)(x)$ and $\varphi(g)(y)$ for $g \in \Pi_{2}$ can be determined just by this combinability condition. This part, reminiscent of the Maskit combination theorem [5] in Kleinian groups, is shown in Section 5 . What is good for this construction is that the whole process can be passed to a 2 -fold lift of $\varphi$.

Moreover the set $R$ of fourteen points in Figure 5 are robust, in the sense that any homomorphism near to $\varphi$ has the same configuration as $R$. Furthermore, if we consider a 2-fold lift $\psi$ of $\varphi$, The set $\pi_{2}^{-1}(R)$ is also robust for $\psi$. This part, shown in Section 7, concludes the proof of the local stability (Theorem 1.17) for $g=2$.

For $g \geq 3$, the group $\Pi_{g}$ is represented as the fundamental group of a tree of groups. Each vertex of the tree has valency either 1 or 3 . For a valency 3 vertex, we have a homomorphism $\varphi \in \mathcal{R}_{\Gamma}$, where $\Gamma$ is the free group on two generators $A$ and $B$. The homeomorphism $a=\varphi(A)$ and $b=\varphi(B)$ has the property that $c(a, b)=1$. This implies that $\varphi$ admits a configuration in Figure 2 right. For this we consider a basic partition $P$ as in Figure 7 . The complementary region consists of four intervals $E_{1}-E_{4}$. The stabilizer of $E_{1}$ is the subgroup $\langle a\rangle$, and we say that $E_{1}$ is the entrance of $\langle a\rangle$ to $P$. Likewise $E_{2}, E_{3}$ and $E_{4}$ are entraces to $P$ of the subgroups $\langle a b\rangle,\langle b\rangle$ and $\langle b a\rangle$, respectively. Compare with Figure 2 right.

\section{Basic partitions}

Let $\Gamma$ be a group with a prescribed finite symmetric generating set $S$.

Definition 4.1. A subset $P$ of $S^{1}$ is called a basic partition (BP) for $\varphi \in \mathcal{R}_{\Gamma}$, if it satisfies the following conditions.

(1) $P$ is a union of finitely many disjoint closed intervals. 
(2) For any $I \sqsubset P$, there exists a unique element $s_{I} \in S$ such that

$$
\varphi\left(s_{I}\right) I=\bigcup_{i=1}^{l} I_{i} \cup \bigcup_{i=1}^{l-1} J_{i},
$$

where $I_{i} \sqsubset P, J_{i} \sqsubset P_{\sharp}$ are distinct intervals and $l \geq 2$. (See Notations 1.19.)

(3) For any $I \sqsubset P$ and $s \in S \backslash\left\{s_{I}\right\}, \varphi(s)(I)$ is a proper subset of some $I^{\prime} \sqsubset P$.

(4) For any $J \sqsubset P_{\sharp}$ and $s \in S$, either $\varphi(s) J \sqsubset P_{\sharp}$ or $\varphi(s) J \subset \operatorname{Int}(P)$.

Example 4.2. The set $P_{\nu}(\nu=1,2)$ in Figure 6 is an example of $\mathrm{BP}$ for homomorphisms $\varphi_{\nu}=\left.\varphi\right|_{\Gamma_{\nu}}$. The set $P$ in Figure 7 is also a BP.

Definition 4.3. For a BP $P$ for $\varphi \in \mathcal{R}_{\Gamma}$ and $l \geq 2$, define inductively $P^{l}=$ $\bigcap_{s \in S \cup\{e\}} \varphi(s) P^{l-1}$, where $P^{1}=P$. Also define $P^{\infty}=\bigcap_{l \in \mathbb{N}} P^{l}$.

Thus $\left\{P^{l}\right\}_{l \in \mathbb{N}}$ is a decreasing sequence of compact subsets, each consisting of finitely many closed intervals. In Example 4.2. if the corresponding homomorphism is onto a Shottky group, then $P^{\infty}$ coincides with the limit set. In general, $P^{\infty}$ is a closed perfect set.

Let us see how $P^{2}$ is obtained from $P$. By (2) and (3) of Definition 4.1, we have

$$
P^{2}=\bigcup_{I \sqsubset P} \varphi\left(s_{I}\right)^{-1}\left(P \cap s_{I}(I)\right) .
$$

That is, any interval $I \sqsubset P$ is divided uniquely as:

$$
I=\bigcup_{i=1}^{l} \varphi\left(s_{I}\right)^{-1}\left(I_{i}\right) \cup \bigcup_{i=1}^{l-1} \varphi\left(s_{I}\right)^{-1}\left(J_{i}\right),
$$

where $\varphi\left(s_{I}^{-1}\right)\left(I_{i}\right) \sqsubset P^{2}, \varphi\left(s_{I}^{-1}\right)\left(J_{i}\right) \sqsubset P_{\sharp}^{2}=\left(P^{2}\right)_{\sharp}$. Any $I^{\prime} \sqsubset P^{2}$ is of the above form $I^{\prime}=\varphi\left(s_{I}\right)^{-1}\left(I_{i}\right)$, and $\varphi\left(s_{I}\right)$ maps $I^{\prime}$ onto $I_{i} \sqsubset P$. For any other $s, \varphi(s)$ maps $I^{\prime}$ onto a proper subset of some $I^{\prime \prime} \sqsubset P^{2}$. On the other hand, $P_{\sharp}^{2}$ is obtained from $P_{\sharp}$ by adding new intervals of the above form $\varphi\left(s_{I}\right)^{-1}\left(J_{i}\right)$. A component of $P_{\sharp}^{2}$ is called level 1 if it is contained in $P_{\sharp}$, and level 2 otherwise. Any level 1 component is mapped by any $\varphi(s)$ onto a component of $P_{\sharp}^{2}$, either to level 1 or to level 2 . As for a level 2 component, we have the following.

(1) A level 2 component $\varphi\left(s_{I}\right)^{-1}\left(J_{i}\right)$ is mapped by $\varphi\left(s_{I}\right)$ onto a level 1 component $J_{i}$, and is mapped by any other $\varphi(s)$ onto an interval contained in the interior of $P^{2}$. Especially no level 2 component is mapped onto a level 2 component.

By these considerations, we have the following lemma.

Lemma 4.4. For a BP $P$ for $\varphi \in \mathcal{R}_{\Gamma}$ and $l \geq 2, P^{l}$ is a $B P$ for $\varphi$.

Let $P\left(\right.$ resp. $\left.P^{\prime}\right)$ be a BP for $\varphi \in \mathcal{R}_{\Gamma}\left(\right.$ resp. $\left.\varphi^{\prime}\right)$. Recall that $P_{*}=P \cap P_{\sharp}$ from Notation 1.19

Definition 4.5. A COP (cyclic order preserving) bijection $\xi: P_{*} \rightarrow P_{*}^{\prime}$ is called a $B P$ equivalence if for any $x, y \in P_{*}$ and $s \in S$, we have

- $[x, y] \sqsubset P$ if and only if $[\xi(x), \xi(y)] \sqsubset P^{\prime}$ and

- $y=\varphi(s) x$ if and only if $\xi(y)=\varphi^{\prime}(s) \xi(x)$.

Lemma 4.6. Let $P$ (resp. $\left.P^{\prime}\right)$ be a BP for $\varphi \in \mathcal{R}_{\Gamma}$ (resp. $\left.\varphi^{\prime}\right)$. Then a BP equivalence $\xi: P_{*} \rightarrow P_{*}^{\prime}$ extends uniquely to a BP equivalence $\xi^{2}: P_{*}^{2} \rightarrow P_{*}^{\prime 2}$. 
Proof. For any $x \in P_{*}^{2} \backslash P_{*}$, there exists a unique element $s \in S$ such that $\varphi(s) x \in P_{*}$. Define $\xi^{2}(x)=\varphi^{\prime}(s)^{-1} \circ \xi \circ \varphi(s)(x)$. It is easy to show that $\xi^{2}$ is in fact a BP equivalence.

Notice that $P^{\infty}=\bigcap_{l \in \mathbb{N}} P^{l}$ is a perfect closed set, $P_{\sharp}^{\infty}=\left(P^{\infty}\right)_{\sharp}$ consists of countably many disjoint closed intervals, and $P_{*}^{\infty}=P^{\infty} \cap P_{\sharp}^{\infty}$ is a countable set. All the three sets are $\varphi(\Gamma)$-invariant.

The next theorem says that if $P$ is a $\mathrm{BP}$ for $\varphi \in \mathcal{R}_{\Gamma}$, then the semiconjugacy class of the homomorphism $\varphi$ is determined by the simple dynamics of $S$ on $P$. A semiconjugacy class is in fact determined by how one or several orbits are located in $S^{1}$ (Proposition 1.6).

TheOREM 4.7. Let $P$ and $P^{\prime}$ be BP's for $\varphi \in \mathcal{R}_{\Gamma}$ and $\varphi^{\prime} \in \mathcal{R}_{\Gamma}$. Then a BP equivalence $\xi: P_{*} \rightarrow P_{*}^{\prime}$ extends uniquely to a $\left(\varphi, \varphi^{\prime}\right)$-equivariant COP bijection $\xi^{\infty}: P_{*}^{\infty} \rightarrow\left(P^{\prime}\right)_{*}^{\infty}$

ProOF. This follows from inductive applications of Lemma 4.6 .

The next lemma plays a key role when we study a $k$-fold lift of a homomorphism. The easy proof is omitted.

LEMma 4.8. Let $P$ be a BP for $\varphi \in \mathcal{R}_{\Gamma}$ and $\psi$ a $k$-fold lift of $\varphi$. Then $\pi_{k}^{-1}(P)$ is a $B P$ for $\psi$.

The lemma joined with Theorem 4.7 says that if $\psi$ is a $k$-fold lift of $\varphi$ which admits a $\mathrm{BP} P$, then the semiconjugacy class of $\psi$ is determined by the dynamics of $\psi(S)$ on $\pi_{k}^{-1}(P)$.

For future purpose, we need to continue to study more about BP's. Especially we have to show that the stabilizer (defined later) of an interval $J \sqsubset P_{\sharp}$ can be determined by a simple algorythm for a certain class of BP's.

Definition 4.9. For any $J \sqsubset P_{\sharp}^{\infty}$, define the level of $J, \operatorname{lev}(J) \in \mathbb{N}$, by $\operatorname{lev}(J)=l$ if and only if $J \subset P_{\sharp}^{l} \backslash P_{\sharp}^{l-1}$.

LEMma 4.10. Let $P$ be a BP for $\varphi \in \mathcal{R}_{\Gamma}$. If $J \sqsubset P_{\sharp}^{\infty}$ satisfies $\operatorname{lev}(J)=l$ for some $l \geq 2$, then there is a unique element $s \in S$ such that $\operatorname{lev}(\varphi(s) J)=l-1$, and for any other $s \in S, \operatorname{lev}(\varphi(s) J)=l+1$.

Proof. For $l=2$, this follows from (1) placed just before Lemma 4.4. The general case can be shown by an easy induction.

Definition 4.11. A labelled directed graph $G(P)$ associated with a BP $P$ for $\varphi \in \mathcal{R}_{\Gamma}$ is defined as follows. The vertices of $G(P)$ are components of $P_{\sharp}$. There is a directed edge from $J_{1}$ to $J_{2}$ with label $s \in S$ (written $J_{1} \stackrel{s}{\rightarrow} J_{2}$ ) if $s=s_{I}$, where $I$ is the component of $P$ right adjacent to $J_{1}$, and $\varphi(s)\left(J_{1}\right)=J_{2}$.

ExAmPLE 4.12. The graph $G\left(P_{1}\right)$ and $G\left(P_{2}\right)$ of the BP's in Figure 6 consists of one cycle, while the graph $G(P)$ for Figure 7 consists of 3 cycles.

Notice that for any vertex $J$ of $G(P)$, there is exactly one edge leaving $J$. However there may be a vertex at which no edges arrive.

Definition 4.13. A BP $P$ for $\varphi \in \mathcal{R}_{\Gamma}$ is called pure, if the graph $G(P)$ consists of disjoint cycles. We allow a period one cycle formed by one vertex and one edge. 
In fact, the pureness does not change if we replace "right adjacent" by "left adjacent" in Definition 4.11, although the direction or labelling of the graph may change. For any BP $P, P^{2}$ can never be pure. The BP's in Examples 4.12 are pure.

Definition 4.14. For $\varphi \in \mathcal{R}_{\Gamma}$ and a subset $A$ of $S^{1}$, the stabilizer of $A$ with respect to $\varphi$, denoted by $\operatorname{Stab}_{\varphi}(A)$, is defined by

$$
\operatorname{Stab}_{\varphi}(A)=\{\gamma \in \Gamma \mid \varphi(\gamma)(A)=A\}
$$

Lemma 4.15. Let $P$ be a pure BP for $\varphi \in \mathcal{R}_{\Gamma}$. Then we have the following.

(1) The group $\Gamma$ is free with symmetrized free generating set $S$ and $\varphi$ is injective.

(2) For any $J \sqsubset P_{\sharp}$, the stabilizer $\operatorname{Stab}_{\varphi}(J)$ is generated by an element written as a cyclically reduced word of $S$.

(3) For any $J \sqsubset P_{\sharp}^{\infty}$ with $\operatorname{lev}(J)=l(l \geq 2), \operatorname{Stab}_{\varphi}(J)$ is generated by an element which has a nonreducing representation $\alpha \beta \alpha^{-1}$ by reduced words of $S$ such that the word length of $\alpha$ is $l-1$ and $\beta$ is cyclically reduced.

Proof. For any $J \sqsubset P_{\sharp}$, assume $\varphi(\gamma)(J)=J$ for some $\gamma \in \Gamma \backslash\{e\}$. Write $\gamma$ as a reduced word in $S: \gamma=s_{m} \cdots s_{2} s_{1}$. For any $1 \leq i \leq m$, let $J_{i}=\varphi\left(s_{i} \cdots s_{1}\right)(J)$. Then we have $\operatorname{lev}\left(J_{i}\right)=1$ for any $i$, that is, $J_{i}$ is a vertex of $G(P)$. In fact, if $\operatorname{lev}\left(J_{i}\right)$ would take the maximal value $l \geq 2$ at some $i$, then by Lemma 4.10, we have $\operatorname{lev}\left(J_{i-1}\right)=\operatorname{lev}\left(J_{i+1}\right)=l-1$ and $s_{i+1}=s_{i}^{-1}$, contrary to the assumption that the word is reduced. Again since the word is reduced and $P$ is pure, we have either of the following.

$$
J \stackrel{s_{1}}{\rightarrow} J_{1} \stackrel{s_{2}}{\rightarrow} \ldots \stackrel{s_{m}}{\rightarrow} J_{m}=J \quad \text { or } J \stackrel{s_{1}}{\leftarrow} J_{1} \stackrel{s_{2}}{\leftarrow} \ldots \stackrel{s_{m}}{\leftarrow} J_{m}=J .
$$

Let $I_{i} \sqsubset P$ be an interval right adjacent to $J_{i}$. Then in the former case $\varphi\left(s_{i+1}\right)$ is always expanding on $I_{i}$, that is, $s_{i+1}=s_{I_{i}}$. This shows that $\varphi(\gamma)$ cannot be the identity. The same is true in the latter case. Points (1) and (2) follows from this, while it is easy to derive (3) from (2).

Finally we shall prepare some terminologies and facts needed for the next section. Let $\Lambda$ be an infinite cyclic subgroup of $\Gamma$ and $\varphi \in \mathcal{R}_{\Gamma}$.

Definition 4.16. Given a closed subset $X$ of $S^{1}$, the set

$$
E_{\varphi}^{\Lambda}(X)=\bigcup\left\{J \sqsubset X_{\sharp} \mid\{e\} \neq \operatorname{Stab}_{\varphi}(J) \subset \Lambda\right\},
$$

is called the entrance of $\Lambda$ to $X$ with respect to $\varphi$.

Definition 4.17. A pair of closed subsets $(Q, E)$ is called a $(\Gamma, \Lambda)$-pair for $\varphi$ if $Q$ is a $\varphi(\Gamma)$-invariant closed perfect set, $E=E_{\varphi}^{\Lambda}(Q)$, and $E$ is a finite disjoint union of closed intervals.

Lemma 4.18. Let $P$ be a pure BP for $\varphi \in \mathcal{R}_{\Gamma}$ and $\Lambda$ an infinite cyclic subgroup of $\Gamma$. Assume $E_{\varphi}^{\Lambda}(P)$ is nonempty. Then $\left(P^{\infty}, E_{\varphi}^{\Lambda}(P)\right)$ is a $(\Gamma, \Lambda)$-pair.

Proof. We only need to show that $E_{\varphi}^{\Lambda}(P)=E_{\varphi}^{\Lambda}\left(P^{\infty}\right)$. That is, if $J \sqsubset P_{\sharp}^{\infty}$ and $\operatorname{Stab}_{\varphi}(J) \subset \Lambda$, then $\operatorname{lev}(J)=1$. But this is clear from Lemma 4.15

\section{Combinations}

This section is divided into three subsections. In the first, we are concerned with a single homomorphism, while in the second, with a pair of homomorphisms.

1. Throughout this subsection, we assume the following. 
Assumption 5.1. (a) The group $G$ is written as an amalgamated product

$$
G=\Gamma_{1} *_{\Lambda} \Gamma_{2},
$$

where $\Lambda$ is an infinite cyclic subgroup.

(b) $\varphi \in \mathcal{R}_{G}$, and $\varphi_{\nu}=\left.\varphi\right|_{\Gamma_{\nu}}$ is injective for $\nu=1,2$.

(c) $\left(Q_{\nu}, E_{\nu}\right)$ is a $\left(\Gamma_{\nu}, \Lambda\right)$-pair for $\varphi_{\nu}, \nu=1,2$.

Denote $\Gamma_{\nu}^{*}=\Gamma_{\nu} \backslash \Lambda$. We make extensive use of the following partition of the group $G$.

$$
\begin{gathered}
G=\bigsqcup_{k \geq 0} G^{k}, \text { where } \\
G^{0}=\Lambda, G^{1}=\Gamma_{1}^{*} \sqcup \Gamma_{2}^{*}, G^{2}=\Gamma_{1}^{*} \Gamma_{2}^{*} \sqcup \Gamma_{2}^{*} \Gamma_{1}^{*}, G^{3}=\Gamma_{1}^{*} \Gamma_{2}^{*} \Gamma_{1}^{*} \sqcup \Gamma_{2}^{*} \Gamma_{1}^{*} \Gamma_{2}^{*}, \cdots .
\end{gathered}
$$

Definition 5.2. The pairs $\left(Q_{1}, E_{1}\right)$ and $\left(Q_{2}, E_{2}\right)$ are called combinable for $\varphi$ if $E_{1}$ and $E_{2}$ alternate in $S^{1}$, that is, $E_{1} \cup E_{2}=S^{1}$ and $\operatorname{Int}\left(E_{1}\right) \cap \operatorname{Int}\left(E_{2}\right)=\emptyset$. In this case we denote $E_{*}=\partial E_{1}=\partial E_{2}$.

We also assume the following in this subsection.

Assumption 5.3. $\mathcal{Q}=\left(\left(Q_{1}, E_{1}\right),\left(Q_{2}, E_{2}\right)\right)$ is a combinable pair for $\varphi$. follows.

We define an (undirected) graph $(\mathbb{V}(\mathcal{Q}), \mathbb{E}(\mathcal{Q})$ ) of the combinable pair $\mathcal{Q}$ as

$$
\begin{gathered}
\mathbb{V}(\mathcal{Q})=\left\{\varphi(g) Q_{\nu} \mid g \in G, \nu=1,2\right\}, \\
\mathbb{E}(\mathcal{Q})=\left\{\left\{v, v^{\prime}\right\} \mid v, v^{\prime} \in \mathbb{V}(\mathcal{Q}), v \neq v^{\prime}, v \cap v^{\prime} \neq \emptyset\right\} .
\end{gathered}
$$

The group $G$ acts naturally on the graph $(\mathbb{V}(\mathcal{Q}), \mathbb{E}(\mathcal{Q}))$ as graph automorphisms via the homomorphism $\varphi$. The rest of this subsection is devoted to the study of properties of the graph $(\mathbb{V}(\mathcal{Q}), \mathbb{E}(\mathcal{Q}))$. Especially we show that the graph $(\mathbb{V}(\mathcal{Q}), \mathbb{E}(\mathcal{Q}))$ is in fact a tree. (It is isomorphic to the Bass-Serre tree associated to the amalgamated product $G=\Gamma_{1} *_{\Lambda} \Gamma_{2}$.)

For $v, w \in \mathbb{V}(\mathcal{Q})$, we denote $v \sim w$ if $\{v, w\} \in \mathbb{E}(\mathcal{Q})$, and say that $v$ and $w$ are adjacent. The indexing set for $Q_{\nu}$ is the group $\mathbb{Z} / 2 \mathbb{Z}$, thus for example $Q_{3}=Q_{1}$, while the indexing set for a group element is $\mathbb{Z}$, thus in general $\gamma_{3} \neq \gamma_{1}$.

Lemma 5.4. We have $Q_{\nu} \sim Q_{\nu+1}$ and $Q_{\nu} \sim \varphi\left(\gamma_{\nu}\right) Q_{\nu+1}$ for any $\gamma_{\nu} \in \Gamma_{\nu}^{*}$. Conversely if $Q_{\nu} \sim v$, then either $v=Q_{\nu+1}$ or $v=\varphi\left(\gamma_{\nu}\right) Q_{\nu+1}$ for some $\gamma_{\nu} \in \Gamma_{\nu}^{*}$.

Proof. Since $Q_{1} \cap Q_{2}=E_{*} \neq \emptyset$, we have $Q_{1} \sim Q_{2}$. Since $Q_{\nu}$ is invariant by $\varphi\left(\Gamma_{\nu}\right)$. we have $Q_{\nu} \cap \varphi\left(\gamma_{\nu}\right) Q_{\nu+1}=\varphi\left(\gamma_{\nu}\right)\left(Q_{1} \cap Q_{2}\right) \neq \emptyset$ for $\gamma_{\nu} \in \Gamma_{\nu}^{*}$. That is, $Q_{\nu} \sim \varphi\left(\gamma_{\nu}\right) Q_{\nu+1}$.

In the rest, we shall show that all the other vertices are not adjacent to $Q_{\nu}$. First we prepare some fundamental facts. See Figure 8.

$$
\begin{gathered}
Q_{\nu} \subset E_{\nu+1} \text { and } Q_{\nu} \cap \operatorname{Int} E_{\nu}=\emptyset, \\
\varphi\left(\gamma_{\nu}\right) E_{\nu} \subset \operatorname{Int} E_{\nu+1} \text { and } \operatorname{Int} \varphi\left(\gamma_{\nu}\right) E_{\nu} \cap Q_{\nu}=\emptyset \text { for any } \gamma_{\nu} \in \Gamma_{\nu}^{*} .
\end{gathered}
$$

For (5.3), recall that $Q_{\nu}$ is assumed to be perfect.

Now for $\gamma_{\nu+1} \in \Gamma_{\nu+1}^{*}$, the vertex $\varphi\left(\gamma_{\nu+1}\right) Q_{\nu}$ is not adjacent to $Q_{\nu}$, since

$$
\varphi\left(\gamma_{\nu+1}\right) Q_{\nu} \subset \varphi\left(\gamma_{\nu+1}\right) E_{\nu+1} \subset \operatorname{Int} E_{\nu} .
$$


FiguRE 8 . The subsets $Q_{\nu}$ should have countably many complementary intervals. Only some of them are drawn in the figure.

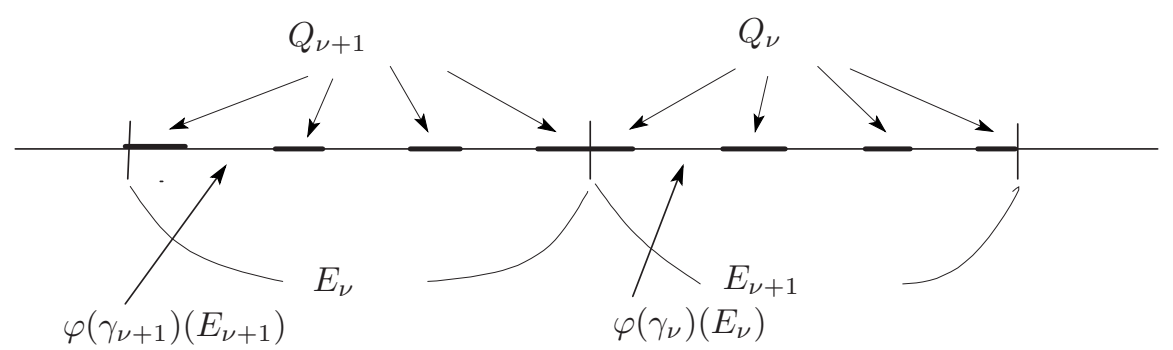

Figure 9. The vertices $a-d$ are $a=Q_{2}, b=Q_{1}, c=\gamma_{1} Q_{2}$ and $d=\gamma_{1} \gamma_{2} Q_{1}$. The actual set $Q_{1}$ is depicted on the circle.

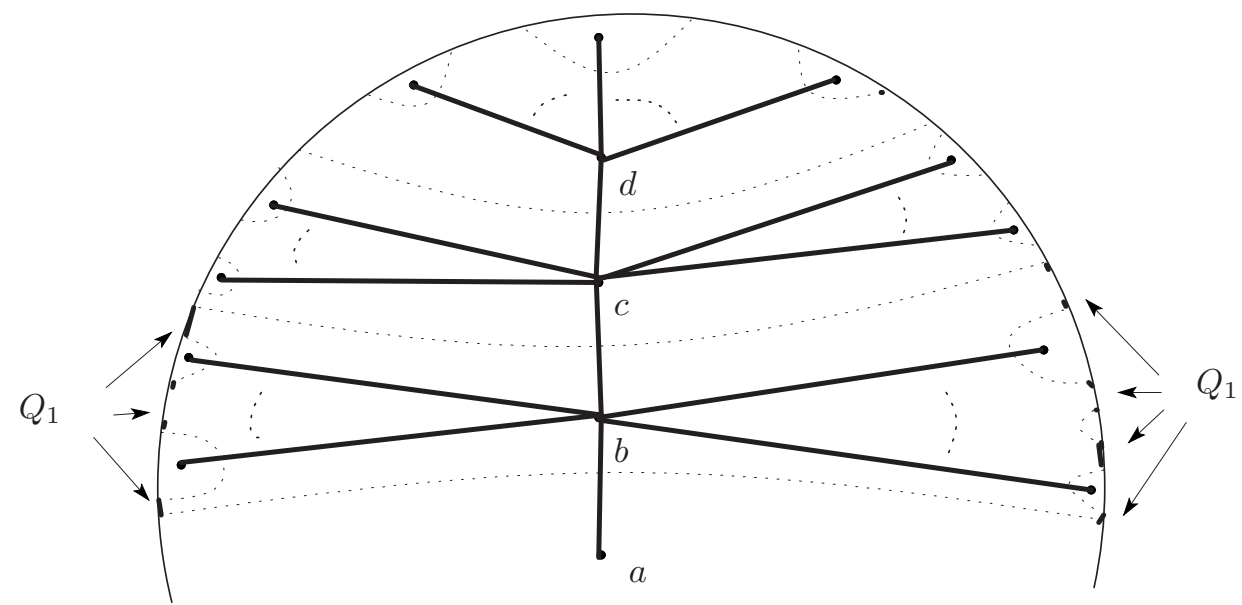

We shall show by induction on $k$ that if $k \geq 2$ and $\gamma_{\nu+i} \in \Gamma_{\nu+i}^{*}(1 \leq i \leq k)$, then

$$
\varphi\left(\gamma_{\nu+k} \cdots \gamma_{\nu+1}\right) Q_{\nu} \subset \operatorname{Int} \varphi\left(\gamma_{\nu+k}\right) E_{\nu+k}
$$

This shows that $\varphi\left(\gamma_{\nu+k} \cdots \gamma_{\nu+1}\right) Q_{\nu}$ is adjacent neither to $Q_{1}$ nor to $Q_{2}$, by virtue of (5.3).

To show (5.4) for $k=2$, notice by (5.2)

$$
\varphi\left(\gamma_{\nu+2} \gamma_{\nu+1}\right) Q_{\nu} \subset \varphi\left(\gamma_{\nu+2} \gamma_{\nu+1}\right) E_{\nu+1} \subset \operatorname{Int} \varphi\left(\gamma_{\nu+2}\right) E_{\nu+2}
$$

For the inductive step,

$$
\varphi\left(\gamma_{\nu+k+1} \gamma_{\nu+k} \cdots \gamma_{\nu+1}\right) Q_{\nu} \subset \varphi\left(\gamma_{\nu+k+1}\right) \operatorname{Int} \varphi\left(\gamma_{\nu+k}\right) E_{\nu+k} \subset \operatorname{Int} \varphi\left(\gamma_{\nu+k+1}\right) E_{\nu+k+1} .
$$

REMARK 5.5. The above proof shows that any vertex $\varphi(g) Q_{\nu}$ is distinct from $Q_{\nu}$ unless $g \in \Gamma_{\nu}$.

See Figure 9 for the graph $(\mathbb{V}(\mathcal{Q}), \mathbb{E}(\mathcal{Q}))$. 
LEMMA 5.6. For any interval $J \sqsubset\left(Q_{\nu}\right)_{\sharp}$ which is distinct from $\varphi\left(\gamma_{\nu}\right) E_{\nu}$ for any $\gamma_{\nu} \in \Gamma_{\nu}$, we have Int $J \cap v=\emptyset$ for any $v \in \mathbb{V}(\mathcal{Q})$ and $\operatorname{Stab}_{\varphi}(J)=\operatorname{Stab}_{\varphi_{\nu}}(J)$.

PRoOF. Any vertex other than $Q_{\nu}$ contained in $E_{\nu+1}$ is contained in $\varphi\left(\gamma_{\nu}\right) E_{\nu}$ for some $\gamma_{\nu} \in \Gamma_{\nu}^{*}$, by virtue of (5.4), showing the first statement. For the last statement, choose an arbitrary element $g \in \operatorname{Stab}_{\varphi}(J)$. Then $g$ leaves $\partial J$ invariant. The set $\partial J$ is contained in $Q_{\nu}$ and disjoint from any other vertex of $\mathbb{V}(\mathcal{Q})$. Therefore $g$ stabilizes the vertex $Q_{\nu}$ in the action of $G$ on the graph. This shows $g \in \Gamma_{\nu}$ by Remark 5.5.

Let us continue the study of the graph $(\mathbb{V}(\mathcal{Q}), \mathbb{E}(\mathcal{Q}))$.

LEMmA 5.7. Let $v, w \in \mathbb{V}(\mathcal{Q})$. If $v \sim w$ and $v=\varphi\left(\gamma_{\nu+k} \cdots \gamma_{\nu+1}\right) Q_{\nu}$ for some $k \geq 1$ and $\gamma_{\nu+i} \in \Gamma_{\nu+i}^{*}$, then either $w=\varphi\left(\gamma_{\nu+k} \cdots \gamma_{\nu+2}\right) Q_{\nu+1}$ or $w=$ $\varphi\left(\gamma_{\nu+k} \cdots \gamma_{\nu+1} \gamma_{\nu}\right) Q_{\nu-1}$ for some $\gamma_{\nu} \in \Gamma_{\nu}^{*}$, and moreover $v \cap w$ is contained in the $\varphi(G)$-orbit of $E_{*}$.

Proof. Recall that the group $G$ acts on the graph $(\mathbb{V}(\mathcal{Q}), \mathbb{E}(\mathcal{Q}))$ as graph automorphisms. Thus if $w \sim \varphi\left(\gamma_{\nu+k} \cdots \gamma_{\nu+1}\right) Q_{\nu}$, then $\varphi\left(\gamma_{\nu+k} \cdots \gamma_{\nu+1}\right)^{-1} w \sim$ $Q_{\nu}$. Therefore either $\varphi\left(\gamma_{\nu+k} \cdots \gamma_{\nu+1}\right)^{-1} w$ is equal to $Q_{\nu+1}$ or $\varphi\left(\gamma_{\nu}\right) Q_{\nu-1}$ for some $\gamma_{\nu} \in \Gamma_{\nu}^{*}$. Since $\varphi\left(\gamma_{\nu+1}\right) Q_{\nu+1}=Q_{\nu+1}$, this shows the first part. An immediate consequence is that $G$ acts transitively on the set of edges $\mathbb{E}(\mathcal{Q})$. That is, there is $g \in G$ which maps $E_{*}=Q_{1} \cap Q_{2}$ onto $v \cap w$, showing the second part.

Definition 5.8. Any vertex $v$ of the graph is written as $v=\varphi\left(\gamma_{\nu+k} \cdots \gamma_{\nu+1}\right) Q_{\nu}$ for $\gamma_{\nu+i} \in \Gamma_{\nu+i}^{*}$. The number $k$ is unique, and is called the distance of $v$.

LEMmA 5.9. (1) We have $\operatorname{Stab}_{\varphi}\left(Q_{\nu}\right)=\Gamma_{\nu}$.

(2) The graph $(\mathbb{V}(\mathcal{Q}), \mathbb{E}(\mathcal{Q}))$ is a tree and $\varphi$ is injective.

(3) For $g \in G$, we have $\varphi(g) E_{*} \cap E_{*} \neq \emptyset$ if and only if $g \in \Lambda$.

Proof. Point (1) is a rephrasing of Remark 5.5. Lemma 5.7 says that any vertex of distance $k(k \geq 1)$ is only adjacent to vertices of distance $k-1$ and $k+1$, and the vertex of distance $k-1$ is unique. This shows that the graph $(\mathbb{V}(\mathcal{Q}), \mathbb{E}(\mathcal{Q}))$ is a tree. To show that $\varphi$ is injective, assume $\varphi(g)=\mathrm{id}$ for some $g \in G$. Then $g$ acts trivially on the graph. Especially $g$ leaves the vertex $Q_{\nu}$ invariant. That is, $g \in \Gamma_{\nu}$ by (1). By the assumption that $\varphi_{\nu}=\left.\varphi\right|_{\Gamma_{\nu}}$ is injective, we get $g=e$, as is required. The if part of (3) is clear. To show the converse, notice that $E_{*}=Q_{1} \cap Q_{2}$. By Lemma 5.4 if $\varphi(g) Q_{\nu} \cap Q_{\nu+1} \neq \emptyset$, then $g \in \Gamma_{\nu+1}$. This holds for each $\nu=1,2$, and thus $g \in \Gamma_{1} \cap \Gamma_{2}=\Lambda$.

Further discussions are necessary for the development of the next subsection.

Definition 5.10. Let $\mathcal{J}_{0}$ be the family of connected components of $E_{1}$ and $E_{2}$, and for $n \geq 1$, let

$$
\mathcal{J}_{n}=\left\{\varphi\left(\gamma_{\nu+n-1} \cdots \gamma_{\nu}\right) I \mid \gamma_{\nu+i} \in \Gamma_{\nu+i}^{*}, I \sqsubset E_{\nu}, \nu=1,2\right\} .
$$

LEMma 5.11. (1) For any $J \in \mathcal{J}_{n}(n \geq 2)$, there are $J_{i} \in \mathcal{J}_{i}(1 \leq i \leq n-1)$ and $\nu$ such that

$$
J \subset \operatorname{Int} J_{n-1} \subset J_{n-1} \subset \cdots \subset \operatorname{Int} J_{1} \subset J_{1} \sqsubset\left(Q_{\nu}\right)_{\sharp},
$$

(2) Any two intervals $J, J^{\prime} \in \mathcal{J}_{n}, n \geq 1$, satisfy either $J=J^{\prime}$ or $J \cap J^{\prime}=\emptyset$. 
Proof. Point (1) is shown inductively using (5.3). Point (2) is clear for $n=1$. (See Figure 8.) The general case can be shown by an induction on $n$ based upon (1).

For a subset $K$ of $G$ and $X$ of $S^{1}$, denote $\varphi(K) X=\cup_{g \in K} \varphi(g) X$.

Definition 5.12. Define a subset $X_{n}$ of $S^{1}$ by $X_{0}=E_{*}$ and for $n \geq 1$, $X_{n}=\varphi\left(G^{n}\right) X_{0}$. Let $X=\bigcup_{n} X_{n}$. (For the definition of $G^{n}$, see (5.1).)

The following easy lemma is useful to clarify an argument in the next subsection.

LEMma 5.13. (1) $X_{n}=\bigcup_{I \in \mathcal{J}_{n}} \partial I$.

(2) $X_{n} \cap X_{m}=\emptyset$ if $n \neq m$.

(3) $X=\varphi(G) E_{*}$.

(4) For any $v \in \mathbb{V}(\mathcal{Q})$ of distance $n(n \geq 0), v \cap X_{m} \neq \emptyset$ if and only if $m=n$ or $m=n+1$.

The following lemma will be used in Section 6 where we consider successive combinations.

Lemma 5.14. Assume there is a subset $E^{\prime} \subset\left(Q_{1}\right)_{\sharp}$ such that $\left(Q_{1}, E^{\prime}\right)$ is a $\left(\Gamma_{1}, \Lambda^{\prime}\right)$-pair for $\varphi_{1}$, where $\Lambda^{\prime}$ is an infinite cyclic subgroup of $\Gamma_{1}$ such that $\Lambda^{\prime} \cap$ $\gamma_{1} \Lambda \gamma_{1}^{-1}=\{e\}$ for any $\gamma_{1} \in \Gamma_{1}$. Then $\left(\operatorname{Cl}\left(\varphi(G)\left(Q_{1} \cup Q_{2}\right)\right)\right.$, $\left.E^{\prime}\right)$ is a $\left(G, \Lambda^{\prime}\right)$-pair for $\varphi$.

Proof. It is clear that

$$
Z:=\operatorname{Cl}\left(\varphi(G)\left(Q_{1} \cup Q_{2}\right)\right)=\operatorname{Cl}\left(\bigcup_{v \in \mathbb{V}(\mathcal{Q})} v\right)
$$

is a $\varphi(G)$-invariant closed perfect set. So what is left is to show that $E^{\prime}=$ $E_{\varphi}^{\Lambda^{\prime}}(Z)$, where by definition $E^{\prime}=E_{\varphi_{1}}^{\Lambda^{\prime}}\left(Q_{1}\right)$. The assumption on $\Lambda^{\prime}$ implies that $E^{\prime} \cap \varphi\left(\gamma_{\nu}\right) E_{\nu}=\emptyset$ for any $\nu$ and $\gamma_{\nu} \in \Gamma_{\nu}^{*}$. By Lemma 5.6, we have $E^{\prime} \subset E_{\varphi}^{\Lambda^{\prime}}(Z)$. To show the converse, assume $J \sqsubset E_{\varphi}^{\Lambda^{\prime}}(Z)$. If $J \sqsubset\left(Q_{1} \cup Q_{2}\right)_{\sharp}$, then clearly we have $J \sqsubset E^{\prime}$. Otherwise $J$ must be contained in $\varphi\left(\gamma_{\nu}\right) I_{\nu} \in \mathcal{J}_{1}$ for some $I_{\nu} \sqsubset E_{\nu}$ and $\gamma_{\nu} \in \Gamma_{\nu}^{*}$. Since $J \sqsubset E_{\varphi}^{\Lambda^{\prime}}(Z)$, there is $g \in \Lambda^{\prime} \backslash\{e\} \subset \Gamma_{1}^{*}$ such that $\varphi(g) J=J$. Then $\varphi(g) \varphi\left(\gamma_{\nu}\right) I_{\nu} \cap \varphi\left(\gamma_{\nu}\right) I_{\nu} \neq \emptyset$. If $\nu=2$, then $\varphi\left(\gamma_{2}\right) I_{2} \subset \operatorname{Int} E_{1}$, while $\varphi(g) \varphi\left(\gamma_{2}\right) I_{2} \subset \operatorname{Int} E_{2}$. A contradiction. If $\nu=1, \varphi(g) \varphi\left(\gamma_{1}\right) I_{1} \in \mathcal{J}_{1}$ since $g \in \Gamma_{1}^{*}$. Then by Lemma $5.11(2), \varphi\left(g \gamma_{1}\right) I_{1}=\varphi\left(\gamma_{1}\right) I_{1}$, and $\gamma_{1}^{-1} g \gamma_{1} \in \Lambda$ by Lemma 5.9 (3). But this is contrary to the assumption on $\Lambda^{\prime}$.

2. In this subsection, we assume the following.

Assumption 5.15. Let $\nu=1,2$ and $i=1,2$.

(a) The group $G$ is just as in Assumption 5.1

(b) Let $\varphi^{i} \in \mathcal{R}_{G}$, and assume $\varphi_{\nu}^{i}=\left.\varphi^{i}\right|_{\Gamma_{\nu}}$ is injective.

(c) Let $\left(Q_{\nu}^{i}, E_{\nu}^{i}\right)$ be a $\left(\Gamma_{\nu}, \Lambda\right)$-pair for $\varphi_{\nu}^{i}$.

(d) The pair $\mathcal{Q}^{i}=\left(\left(Q_{1}^{i}, E_{1}^{i}\right),\left(Q_{2}^{i}, E_{2}^{i}\right)\right)$ is combinable for $\varphi^{i}$.

(e) There is a COP bijection $\xi: Q_{1, *}^{1} \cup Q_{2, *}^{1} \rightarrow Q_{1, *}^{2} \cup Q_{2, *}^{2}$ such that $\xi\left(Q_{\nu, *}^{1}\right)=$ $Q_{\nu, *}^{2}$ and the restrictions $\xi_{\nu}=\left.\xi\right|_{Q_{\nu, *}^{1}}: Q_{\nu, *}^{1} \rightarrow Q_{\nu, *}^{2}$ is $\left(\varphi_{\nu}^{1}, \varphi_{\nu}^{2}\right)$-equivariant. 
Our purpose is to show that $\xi$ extends to a $\left(\varphi^{1}, \varphi^{2}\right)$-equivariant COP bijection from the saturation $\varphi^{1}(G)\left(Q_{1, *}^{1} \cup Q_{2, *}^{1}\right)$ to $\varphi^{2}(G)\left(Q_{1, *}^{2} \cup Q_{2, *}^{2}\right)$ (Theorem 5.17). The proof is by two steps: the first step is the following Lemma. Let $\mathcal{J}_{n}^{i}, X_{n}^{i}$ and $X^{i}$ be defined as in Definitions 5.10 and 5.12 for $\varphi^{i}$.

Lemma 5.16. The map $\xi$ extends to a COP bijection

$$
\hat{\xi}: Q_{1, *}^{1} \cup Q_{2, *}^{1} \cup X^{1} \rightarrow Q_{1, *}^{2} \cup Q_{2, *}^{2} \cup X^{2}
$$

which is $\left(\varphi^{1}, \varphi^{2}\right)$-equivariant as a map from $X^{1}$ to $X^{2}$.

Proof. Recall that $X^{i}=\varphi_{i}(G) E_{*}^{i}$. The map $\xi$ extends to $X^{1}$ by the $\left(\varphi^{1}, \varphi^{2}\right)$ equivariance. Namely, given $x \in X^{1}$, choose $g \in G$ and $x_{0} \in E_{*}^{1}$ such that $x=$ $\varphi^{1}(g) x_{0}$, and define $\hat{\xi}(x)=\varphi^{2}(g) \xi\left(x_{0}\right)$. The map $\hat{\xi}$ is a well defined bijection since by Lemma 5.9 (3), $\operatorname{Stab}_{\varphi^{i}}\left(E_{*}^{i}\right) \subset \Lambda$, and $\left.\xi\right|_{E_{*}^{1}}$ is $\left(\left.\varphi^{1}\right|_{\Lambda},\left.\varphi^{2}\right|_{\Lambda}\right)$-equivariant. Notice also that $\hat{\xi}$ coincides with the original $\xi$ on $X_{1}^{1} \subset Q_{1, *}^{1} \cup Q_{2, *}^{1}$ by the $\left(\varphi_{\nu}^{1}, \varphi_{\nu}^{2}\right)$ equivariance of $\xi_{\nu}$, and $X_{n}^{1}(n \geq 2)$ is disjoint from $Q_{1, *}^{1} \cup Q_{2, *}^{1}$. Therefore we only need to show that $\hat{\xi}$ is COP.

We shall prove that $\hat{\xi}$ is COP on $Q_{1, *}^{1} \cup Q_{2, *}^{1} \cup \bigcup_{0 \leq i \leq n} X_{i}^{1}$ by an induction on $n$. This is sufficient since $X^{1}=\bigcup_{n} X_{n}^{1}$. For $n=1$, this is true by the assumption since $X_{1}^{1} \subset Q_{1, *}^{1} \cup Q_{2, *}^{1}$. To show it for $n+1$, choose an arbitrary open interval

$$
\text { Int } I \sqsubset S^{1} \backslash\left(Q_{1, *}^{1} \cup Q_{2, *}^{1} \cup \bigcup_{0 \leq i \leq n} X_{i}^{1}\right)
$$

such that $\operatorname{Int} I \cap X_{n+1}^{1} \neq \emptyset$. Clearly we only have to show that $\hat{\xi}$ is COP on

$$
I \cap\left(Q_{1, *}^{1} \cup Q_{2, *}^{1} \cup \bigcup_{0 \leq i \leq n+1} X_{i}^{1}\right),
$$

where $I$ is the closure of $\operatorname{Int} I$. Now any point of $\operatorname{Int} I \cap X_{n+1}^{1}$ is an endpoint of some interval of $\mathcal{J}_{n+1}^{1}$, and by Lemma 5.11 we have $I \in \mathcal{J}_{n}^{1}$. This shows

$$
I \cap\left(Q_{1, *}^{1} \cup Q_{2, *}^{1} \cup \bigcup_{0 \leq i \leq n+1} X_{i}^{1}\right)=I \cap\left(X_{n}^{1} \cup X_{n+1}^{1}\right) .
$$

Furthermore $I=\varphi^{1}(g) J$ for some $J \in \mathcal{J}_{0}^{1}$ and $g \in G^{n}$.

Finally since we have defined

$$
\left.\hat{\xi}\right|_{I \cap\left(X_{n}^{1} \cup X_{n+1}^{1}\right)}=\left(\left.\varphi^{2}(g)\right|_{\xi(J) \cap\left(X_{0}^{2} \cup X_{1}^{2}\right)}\right) \circ\left(\left.\xi\right|_{J \cap\left(X_{0}^{1} \cup X_{1}^{1}\right)} \circ\left(\left.\varphi^{1}\left(g^{-1}\right)\right|_{I \cap\left(X_{n}^{1} \cup X_{n+1}^{1}\right)}\right),\right.
$$

and all the maps on the RHS is COP, the map $\left.\hat{\xi}\right|_{I \cap\left(X_{n}^{1} \cup X_{n+1}^{1}\right)}$ is COP, as is required.

Theorem 5.17. Under Assumption [5.15, the COP bijection

$$
\xi: Q_{1, *}^{1} \cup Q_{2, *}^{1} \rightarrow Q_{1, *}^{2} \cup Q_{2, *}^{2}
$$

extends uniquely to a $\left(\varphi^{1}, \varphi^{2}\right)$-equivariant COP bijection

$$
\hat{\xi}: \varphi^{1}(G)\left(Q_{1, *}^{1} \cup Q_{2, *}^{1}\right) \rightarrow \varphi^{2}(G)\left(Q_{1, *}^{2} \cup Q_{2, *}^{2}\right) .
$$

Proof. Recall that

$$
\varphi^{1}(G)\left(Q_{1}^{1} \cup Q_{2}^{1}\right)=\bigcup\left\{v \mid v \in \mathbb{V}\left(\mathcal{Q}^{1}\right)\right\},
$$


where $v=\varphi^{1}(g) Q_{\nu}^{1}$ for some $g \in G$ and $\nu$. Denote $v_{*}=\varphi^{1}(g) Q_{\nu, *}^{1}$. Define $\hat{\xi}$ on each $v_{*}$ by the $\left(\varphi^{1}, \varphi^{2}\right)$-equivariance. This is well defined because $\xi$ is $\left(\varphi_{\nu}^{1}, \varphi_{\nu}^{2}\right)$ equivariant on $Q_{\nu, *}^{1}$ and $\operatorname{Stab}_{\varphi^{i}}\left(Q_{\nu}^{i}\right)=\Gamma_{\nu}$ by Lemma 5.9 (1). Of course the map $\hat{\xi}$ is COP on each $v_{*}$. The map $\hat{\xi}$ coincides with the one defined in Lemma 5.16 on $v_{*} \cap X^{1}$. The proof is complete by Lemma 5.16.

3. Let $\nu=1,2$ and $i=1,2$. Assume the following.

(a) The group $G$ is just as in Assumption 5.1]

(b) Let $\varphi^{i} \in \mathcal{R}_{G}$, and denote $\varphi_{\nu}^{i}=\left.\varphi^{i}\right|_{\Gamma_{\nu}}$.

(c) Let $P_{\nu}^{i}$ is a pure BP for $\varphi_{\nu}^{i}$, with $E_{\nu}^{i}$ the entrance of $\Lambda$ to $P_{\nu}^{i}$.

(d) The pairs $\left(P_{1}^{i}, E_{1}^{i}\right)$ and $\left(P_{2}^{i}, E_{2}^{i}\right)$ are combinable in the sense that $E_{1}^{i}$ and $E_{2}^{i}$ are alternating in $S^{1}$.

(e) There is a COP bijection $\xi: P_{1, *}^{1} \cup P_{2, *}^{1} \rightarrow P_{1, *}^{2} \cup P_{2, *}^{2}$ such that $\left.\xi\right|_{P_{\nu, *}^{1}}$ is a BP equivalence from $P_{\nu, *}^{1}$ onto $P_{\nu, *}^{2}$.

Joining Theorems 4.7 and 5.17, we get the following.

TheOREM 5.18. Under the above assumption, $\varphi^{1}$ and $\varphi^{2}$ are semiconjugate.

Notice that the set $R$ of fourteen points in Figure 5 is equal to $P_{1, *}^{1} \cup P_{2, *}^{1}$ for the homomorphism (here denoted $\varphi^{1}$ ) in $\mathcal{R}_{\Pi_{2}}$ with $e u\left(\varphi^{1}\right)=2$. Thus the above theorem says that any homomorphisms which admit the same configuration $R$ are mutually semiconjugate. This, together with the robustness of $R$ (discussed in Section 7), implies the local stability of $\varphi^{1}$. Furthermore a 2 -fold lift of $\varphi^{1}$ is also shown to be locally stable.

\section{Trees of groups}

Definition 6.1. A tree of groups is a finite tree $\mathcal{T}=(\mathcal{V}, \mathcal{E})$ such that (1) to each vertex $v \in \mathcal{V}$ (resp. edge $e \in \mathcal{E}$ ) is associated a group $\Gamma_{v}\left(\right.$ resp. $\left.\Lambda_{e}\right)$,

(2) and if $v \in V$ is an end point of $e \in \mathcal{E}$, then a monomorphism $\iota_{e, v}: \Lambda_{e} \rightarrow \Gamma_{v}$ is assigned.

The fundamental group $G(\mathcal{T})$ of a tree $\mathcal{T}$ of groups is the group generated by $\Gamma_{v}$ and $\Lambda_{e}(v \in \mathcal{V}, e \in \mathcal{E})$ subject to the relation $\lambda=\gamma$ whenever $\lambda \in \Lambda_{e}, \gamma \in \Gamma_{v}, v$ is an end point of $e$, and $\iota_{e, v}(\lambda)=\gamma$.

EXAmple 6.2. Consider the closed oriented surface $\Sigma_{g}$ of genus $g$. Divide $\Sigma_{g}$ by circles into once puctured tori and pairs of pants. Embed a tree in $\Sigma_{g}$ as in Figure 10 top. Then the fundamental groups $\Gamma_{i}$ of subsurfaces $\Sigma_{i}$ and the fundamental groups $\Lambda_{j}$ of circles $C_{j}$ are considered to be subgroups of the fundamental group $\Pi_{g}$ of the total surface, the base points being taken on the tree. This yields a tree of groups as in Figure 10 bottom whose fundamental group is isomorphic to $\Pi_{g}$.

Throughout this section we work under the following assumption.

Assumption 6.3. (a) The group $G=G(\mathcal{T})$ is the fundamental group of a tree $\mathcal{T}=(\mathcal{V}, \mathcal{E})$ of groups.

(b) The vertex group $\Gamma_{v}$ admits a finite symmetric generating set $S_{v}$, and the edge group $\Lambda_{e}$ is infinite cyclic.

(c) If $e$ and $e^{\prime}$ are distinct edges starting at a vertex $v$, then $\Lambda_{e} \cap \lambda_{v} \Lambda_{e^{\prime}} \lambda_{v}^{-1}=\{e\}$ for any $\lambda_{v} \in \Gamma_{v}$. 
FigURE 10.
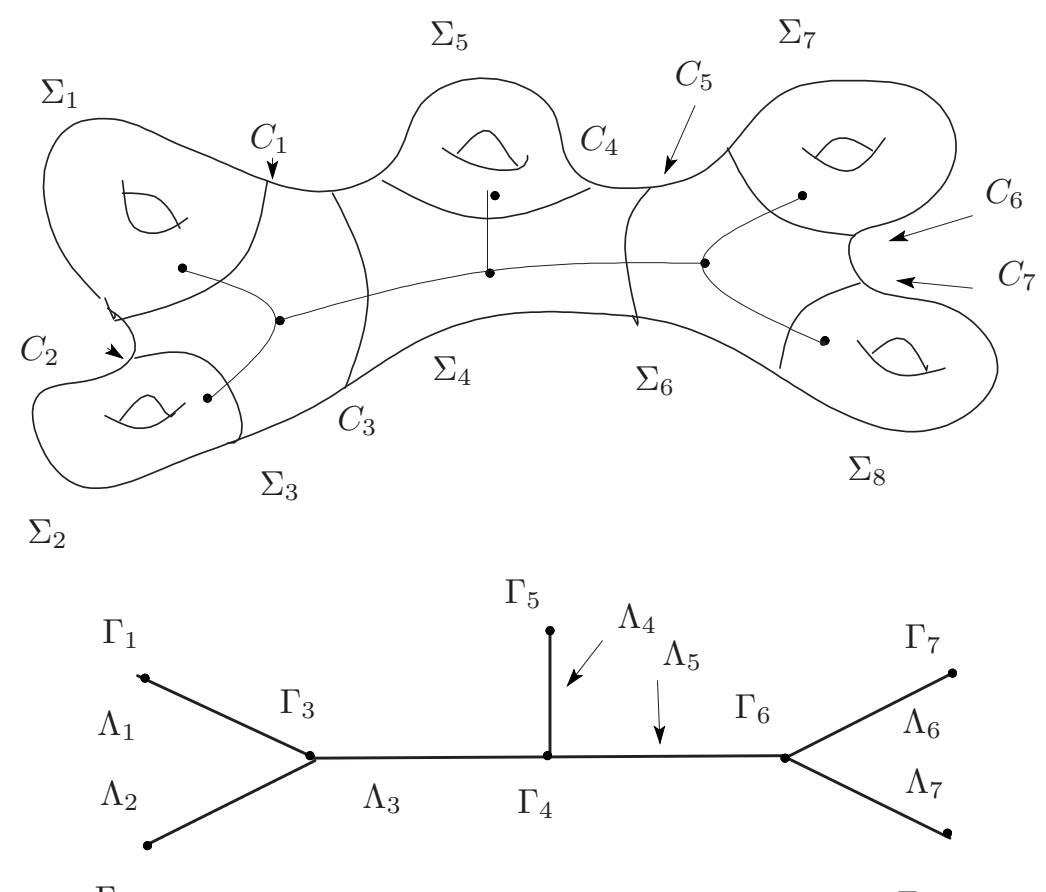

$\Gamma_{2}$

$\Gamma_{8}$

(d) There are two homomorphisms $\varphi^{i} \in \mathcal{R}_{G}, i=1,2$. We denote $\varphi_{v}^{i}$ the restriction of $\varphi^{i}$ to the vertex group $\Gamma_{v}$.

(e) For each vertex group $\Gamma_{v}$, there is a pure $\mathrm{BP} P_{v}^{i}$ for $\varphi_{v}^{i}$ with repect to the generating set $S_{v}$.

(f) If $v$ is an end point of $e$, then there is an entrance, denoted $E_{v, e}^{i}$, of $\Lambda_{e}$ to $P_{v}^{i}$ with respect to $\varphi_{v}^{i}$. Put $Q_{v}^{i}=P_{v}^{i, \infty}$.

Then $\left(Q_{v}^{i}, E_{v, e}^{i}\right)$ is a $\left(\Gamma_{v}, \Lambda_{e}\right)$-pair for $\varphi_{v}^{i}$ by Lemma 4.18 ,

(g) If $v$ and $v^{\prime}$ are two end points of $e$, then $\left(Q_{v}^{i}, E_{v, e}^{i}\right)$ and $\left(Q_{v^{\prime}}^{i}, E_{v^{\prime}, e}^{i}\right)$ form a combinable pair. Denote the finite set $E_{e, *}^{i}=E_{v, e}^{i} \cap E_{v^{\prime}, e}^{i}$.

The set $P_{*}^{i}=\bigcup_{v \in \mathcal{V}} P_{v, *}^{i}$ is called the basic configuration (abbreviated $\mathrm{BC})$ of $G=G(\mathcal{T})$ for $\varphi^{i}$. A COP bijection $\xi: P_{*}^{1} \rightarrow P_{*}^{2}$ is called a $B C$ equivalence if $\xi\left(P_{v, *}^{1}\right)=P_{v, *}^{2}$ and $\left.\xi\right|_{P_{v, *}^{1}}$ is a $\mathrm{BP}$ equivalence from $P_{v, *}^{1}$ to $P_{v, *}^{2}$ for each $v \in \mathcal{V}$.

(h) There is a $\mathrm{BC}$ equivalence $\xi: P_{*}^{1} \rightarrow P_{*}^{2}$.

For our purpose, the following example of $\mathrm{BC}$ is the most important.

EXAMPLE 6.4. Cosider a Fuchsian representation of the surface group $\Pi_{5}$ in Figure 10. Choose a lift $\widetilde{T}$ of the tree $T$ embedded in the surface to the universal covering space $\mathbb{D}$. See Figure 11 . The lift of the curve $C_{j}$ to $\mathbb{D}$ which intersects $\widetilde{T}$ is denoted by $\widetilde{C}_{j}$. The edge group $\Lambda_{j}$ is the stabilizer of $\widetilde{C}_{j}$. As for the vertex group $\Gamma_{i}$, its convex core (of the limit set) is contained in the region $\Sigma_{i}^{\prime}$ depicted in Figure 11. 


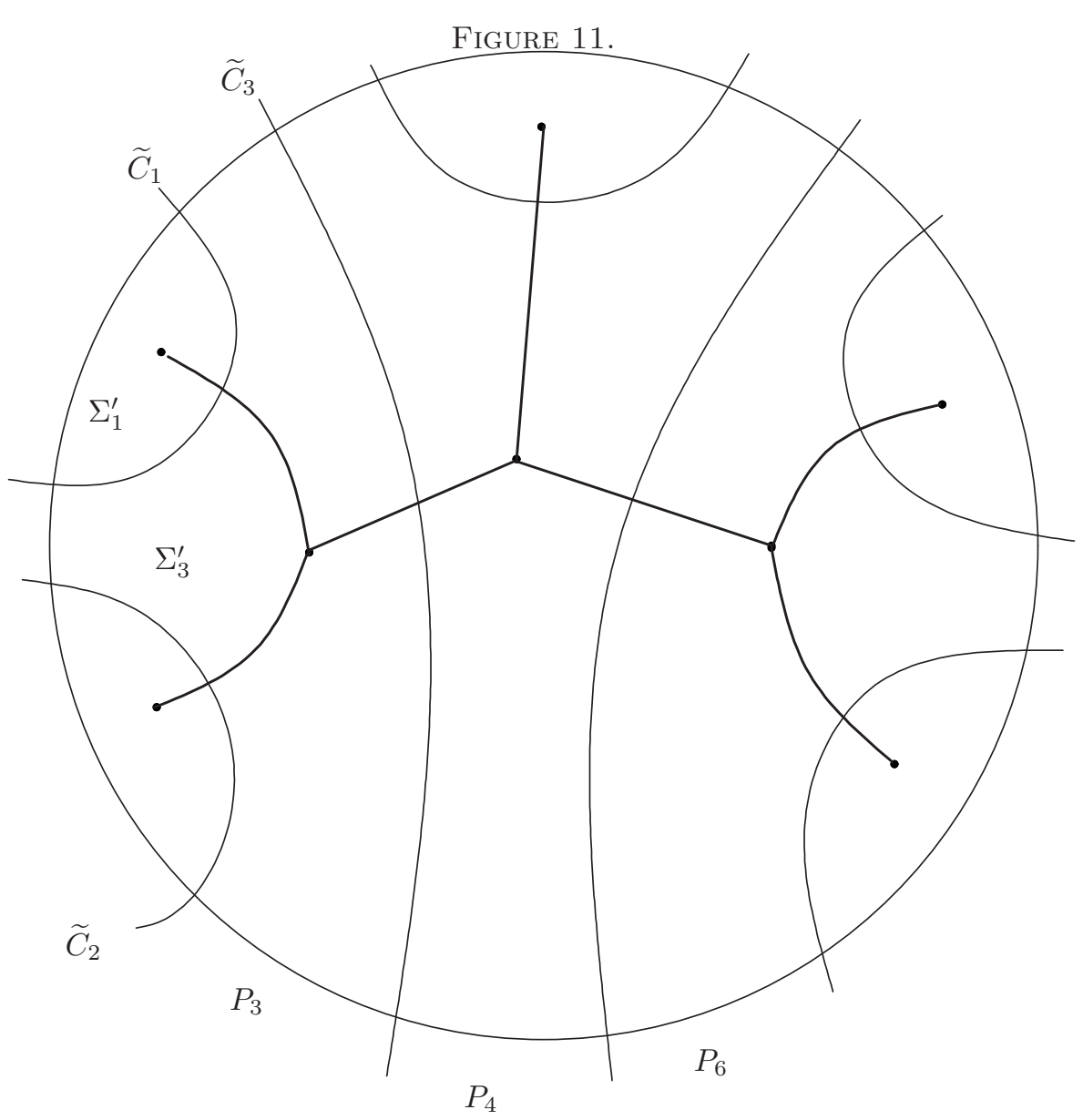

For a vertex of valency 1 , the vertex group is generated by two hyperbolic motions $a$ and $b$ such that $\tau([\widetilde{a}, \widetilde{b}])=1$. So it has a BP as in Figure 6 . For a vertex of valency 3 , generators $a, b$ of the vertex group satisfies $c(a, b)=1$, and it has a BP as in Figure 7 . The BP $P_{1}$ (resp. $P_{3}$ ) corresponding to the vertex group $\Gamma_{1}$ (resp. $\left.\Gamma_{3}\right)$ is depicted in Figure 12. The BC of $\Pi_{5}$ consists of 50 points and satisfies all the conditions of Definition 6.3.

The following lemma is straightforward.

Lemma 6.5. If $P_{*}^{1}$ is a $B C$ for $\varphi^{1} \in \mathcal{R}_{G}$, where $G=G(\mathcal{T})$ is the fundamental group of a tree $\mathcal{T}$, and if $\psi^{1}$ is a $k$-fold lift of $\varphi^{1}$ for some $k \geq 2$, then $\pi_{k}^{-1}\left(P_{*}^{1}\right)$ is a $B C$ for $\psi^{1}$.

Before stating the main theorem of this section, we prepare a lemma. By Theorem 4.7 the BP equivalence $\left.\xi\right|_{P_{v, *}^{1}}: P_{v, *}^{1} \rightarrow P_{v, *}^{2}$ extends to a $\left(\varphi_{v}^{1}, \varphi_{v}^{2}\right)$-equivariant COP bijection $\hat{\xi}_{v}: Q_{v, *}^{1} \rightarrow Q_{v, *}^{2}$ for each vertex $v$. Notice that $Q_{v, *}^{i}=\varphi^{i}\left(\Gamma_{v}\right) P_{v, *}^{i}$. $\hat{\xi}_{v}$.

Lemma 6.6. There is a COP bijection $\hat{\xi}: \bigcup_{v} Q_{v, *}^{1} \rightarrow \bigcup_{v} Q_{v, *}^{2}$ such that $\left.\hat{\xi}\right|_{Q_{v, *}^{1}}=$ 


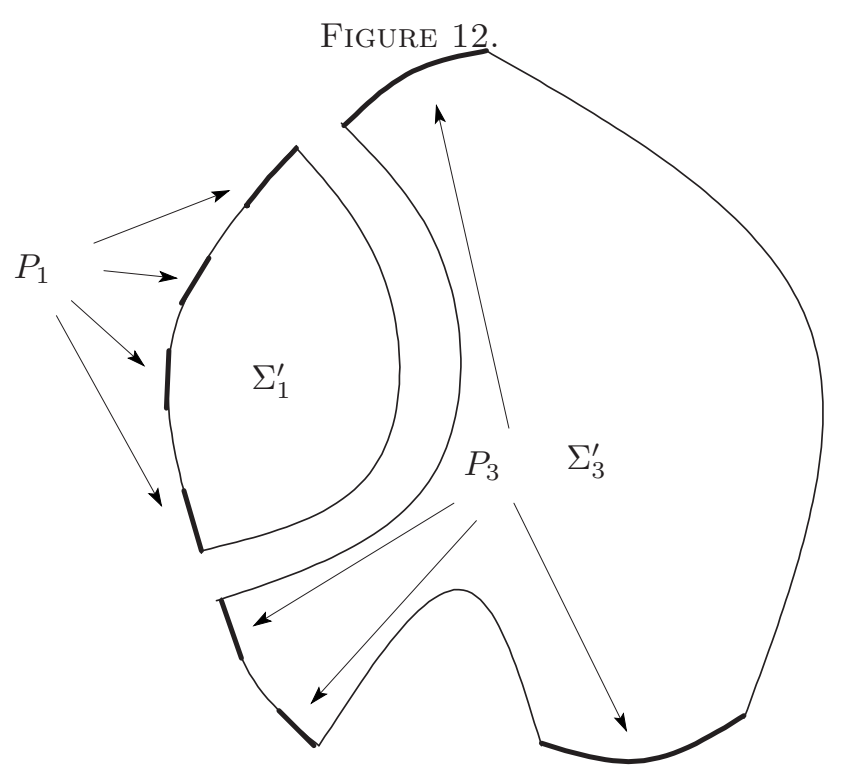

Proof. If $v, v^{\prime}$ are distinct vertices, then $P_{v}^{i} \cap P_{v^{\prime}}^{i} \subset P_{*}^{i}$. In fact, if $v, v^{\prime}$ are adjacent, this follows from (g). If not, $P_{v^{\prime}}^{i}$ is contained in Int $E_{v, e}^{i}$, where $e$ is the edge that starts at $v$ and tends toward the direction of $v^{\prime}$, which implies $P_{v}^{i} \cap P_{v^{\prime}}^{i}=\emptyset$. Since $Q_{v, *}^{i} \subset P_{v}^{i}$, the lemma follows from the fact that both $\xi: P_{*}^{1} \rightarrow P_{*}^{2}$ and $\hat{\xi}_{v}: Q_{v, *}^{1} \rightarrow Q_{v, *}^{2}$ are COP bijections.

Theorem 6.7. The BC-equivalence $\xi: P_{*}^{1} \rightarrow P_{*}^{2}$ extends to a $\left(\varphi^{1}, \varphi^{2}\right)$-equivariant COP bijection $\hat{\xi}: \varphi^{1}(G) P_{*}^{1} \rightarrow \varphi^{2}(G) P_{*}^{2}$.

Proof. The proof is by an induction on the number $n$ of vertices of $\mathcal{T}$. If $n=2$, this is just Theorem 5.17 Given $\mathcal{T}$, delete a vertex $v$ of valency 1 and the edge $e$ which starts at $v$. Denote the resultant subtree by $\mathcal{T}^{\prime}$ and the other end point of $e$ by $v^{\prime}$. Then the group $G=G(\mathcal{T})$ can be written as an amalgamated product:

$$
G=G\left(\mathcal{T}^{\prime}\right) *_{\Lambda_{e}} \Gamma_{v}
$$

Let

$$
Q^{\prime i}=\varphi^{i}\left(G\left(\mathcal{T}^{\prime}\right)\right)\left(\bigcup_{v \in \mathcal{T}^{\prime}} Q_{v}^{i}\right)
$$

Then $\left(Q^{\prime i}, E_{v^{\prime}, e}^{i}\right)$ is shown to be a $\left(G\left(\mathcal{T}^{\prime}\right), \Lambda_{e}\right)$-pair by virtue of Assumption 6.3 (c) and successive use of Lemma 5.14 Clearly the pair $\left(Q^{\prime i}, E_{v^{\prime}, e}^{i}\right)$ is combinable with the $\left(\Gamma_{v}, \Lambda_{e}\right)$-pair $\left(Q_{v}^{i}, E_{v, e}^{i}\right)$. On the other hand, by the induction hypothesis, $\xi$ has an $G\left(\mathcal{T}^{\prime}\right)$-equivariant extension $\xi^{\prime}: Q_{*}^{\prime 1} \rightarrow Q_{*}^{\prime 2}$. Moreover $\xi^{\prime}$ and $\xi_{v}$ satisfy point (e) of Assumption 5.15. The proof is complete by Theorem 5.17

\section{Robust basic configurations}

Again let $G=G(\mathcal{T})$ be the fundamental group of a tree $\mathcal{T}$ of groups. Assume that $\varphi^{1} \in \mathcal{R}_{G}$ satisfies Assumption 6.3 for $\nu=1$, and let $P_{*}^{1}$ be the associated BC. 
Recall that for each vertex $v$ of $\mathcal{T}$ and $l \geq 2,\left(P_{v}^{1}\right)^{l}$ is the BP for $\Gamma_{v}$ derived from the BP $P_{v}^{1}$. (Definition 4.3). Denote $\left(P^{1}\right)_{*}^{l}=\bigcup_{v}\left(P_{v}^{1}\right)_{*}^{l}$.

For each point $x \in P_{*}^{1}$, the stabilizer $\operatorname{Stab}_{\varphi^{1}}(x)$ is infinite cyclic by Lemma 4.15. Lemma 5.9 and a repeated use of Lemma 5.6. Denote by $x_{l}^{+}$(resp. $x_{l}^{-}$) the point in $\left(P^{1}\right)_{*}^{l}$ right (resp. left) adjacent to $x$.

Definition 7.1. The BC $P_{*}^{1}$ is called robust if for any point $x \in P_{*}^{1}$ and any big $l$, one of the generators of $\varphi^{1}\left(\operatorname{Stab}_{\varphi^{1}}(x)\right)$ maps the interval $\left[x_{l}^{-}, x_{l}^{+}\right]$into a proper subinterval of it.

Lemma 7.2. For a homomorphism $\varphi^{1} \in \mathcal{R}_{\Pi_{g}}$ with eu $\left(\varphi^{1}\right)=2 g-2(g \geq 2)$, the $B C$ given by Examples 6.2 and 6.4 is robust.

Proof. If we choose a Fuchsian representation as a model of $\varphi^{1}$, then any point of the $\mathrm{BC}$ is a fixed point of a hyperbolic motion. Any representation $\varphi^{1}$ with $e u\left(\varphi^{1}\right)=2 g-2$ is semiconjugate to the Fuchsian representation by [7], showing the lemma.

Finally we have the following theorem.

TheOREM 7.3. Assume that $\varphi^{1}$ admits a robust $B C P_{*}^{1}$. Then there is a neighbourhood $\mathcal{U}$ of $\varphi^{1}$ in $\mathcal{R}_{G}$ such that if $\varphi^{2} \in \mathcal{U}, \varphi^{2}$ admits a $B C P_{*}^{2}$ and a $B C$ equivalence $\xi: P_{*}^{1} \rightarrow P_{*}^{2}$.

Proof. Choose $l$ large enough so that the condition of Definition 7.1 is met by all the points $x$ in $P_{*}^{1}$ and that the intervals $\left[x_{l}^{-}, x_{l}^{+}\right]$'s are disjoint. Let $g_{x}$ be the generator of $\operatorname{Stab}_{\varphi^{1}}(x)$ such that

$$
\varphi^{1}\left(g_{x}\right)\left[x_{l}^{-}, x_{l}^{+}\right] \subset \operatorname{Int}\left[x_{l}^{-}, x_{l}^{+}\right] .
$$

Choose a neighbourhood $\mathcal{U}$ of $\varphi^{1}$ so that for any $\varphi^{2} \in \mathcal{U}$ and $x \in P_{*}^{1}$, we have

$$
\varphi^{2}\left(g_{x}\right)\left[x_{l}^{-}, x_{l}^{+}\right] \subset \operatorname{Int}\left[x_{l}^{-}, x_{l}^{+}\right] .
$$

Let $\xi(x)$ be the leftmost point in $\operatorname{Fix}\left(\varphi^{2}\left(g_{x}\right)\right) \cap\left[x_{l}^{-}, x_{l}^{+}\right]$. Then the set

$$
P_{*}^{2}=\left\{\xi(x) \mid x \in P_{*}^{1}\right\}
$$

forms a $\mathrm{BC}$ for $\varphi^{2}$, and the map $\xi$ is a $\mathrm{BC}$ equivalence. In fact, it is easy to see that for any vertex $v$,

$$
P_{v, *}^{2}=\left\{\xi(x) \mid x \in P_{v, *}^{1}\right\}
$$

is a $\mathrm{BP}$ for $\left.\varphi^{2}\right|_{\Gamma_{v}}$, because we have assumed that $P_{v, *}^{1}$ is a pure BP.

Joining this theorem with Lemma 6.5 and Theorem 6.7 we get the following corollary, which conclude the proof of Theorem 1.17

COROLlary 7.4. If $\varphi^{1} \in \mathcal{R}_{G}$ admits a robust $B C$, and $\psi^{1} \in \mathcal{R}_{G}$ is a $k$-fold lift of $\varphi^{1}(k \geq 1)$, then $\psi^{1}$ is locally stable.

Proof. Let $P_{*}^{1}$ be a robust $\mathrm{BC}$ for $\varphi^{1}$. Then clearly $\pi_{k}^{-1}\left(P_{*}^{1}\right)$ is a robust $\mathrm{BC}$ for $\psi^{1}$. By Theorem 7.3 , there is a BC $\widetilde{P}_{*}^{2}$ for any $\psi^{2}$ sufficiently near to $\psi^{1}$ and a BC equivalence $\xi: \pi_{k}^{-1}\left(P_{*}^{1}\right) \rightarrow \widetilde{P}_{*}^{2}$. By Theorem 6.7 the BC equivalence $\xi$ extends to a $\left(\psi^{1}, \psi^{2}\right)$-equivariant COP map $\hat{\xi}: \psi^{1}(G)\left(\pi_{k}^{-1}\left(P_{*}^{1}\right)\right) \rightarrow \psi^{2}(G)\left(\widetilde{P}_{*}^{2}\right)$. The map $\hat{\xi}$ extends to a $\left(\psi^{1}, \psi^{2}\right)$-equivariant semiconjugacy. 


\section{Appendix A: The proof of Proposition 1.4}

We shall show that the semiconjugacy as defined in Definition 1.3 is an equivalence relation in $\mathcal{R}_{G} \backslash \mathcal{R}_{G}^{*}$. All that need proof is the reflexiveness. Let $\varphi^{1}, \varphi^{2} \in$ $\mathcal{R}_{G} \backslash \mathcal{R}_{G}^{*}$. Assume there is a degree one monotone map $h: S^{1} \rightarrow S^{1}$ such that

$$
\varphi^{2}(g) \circ h=h \circ \varphi^{1}(g), \quad \forall g \in G .
$$

Since $\varphi^{i} \in \mathcal{R}_{G} \backslash \mathcal{R}_{G}^{*}, h$ is not a constant map. Let $\widetilde{h}: \mathbb{R} \rightarrow \mathbb{R}$ be a lift of $h$ as in Definition [1.2. Notice that such a lift $\tilde{h}$ is unique up to the composition with $T^{n}$, since the map $h$ is nonconstant. (This is why we divide the definition of semiconjugacy into two parts.) Define $\widetilde{h}^{\diamond}: \mathbb{R} \rightarrow \mathbb{R}$ by

$$
\widetilde{h}^{\diamond}(y)=\inf \{x \in \mathbb{R} \mid \tilde{h}(x)=y\} .
$$

Clearly $\widetilde{h}^{\diamond}$ commutes with $T$, and there is a degree one monotone map $h^{\diamond}: S^{1} \rightarrow S^{1}$ such that $h^{\diamond} \circ \pi=\pi \circ \widetilde{h}^{\diamond}$. The well-definedness of $h^{\diamond}$ is garanteed by the uniqueness of $\widetilde{h}$. Moreover if $h, h^{\prime}$ and $h^{\prime} \circ h$ are nonconstant monotone degree one maps, then we have

$$
\left(h^{\prime} \circ h\right)^{\diamond}=h^{\diamond} \circ\left(h^{\prime}\right)^{\diamond} .
$$

Thus (8.1) implies that

$$
h^{\diamond} \circ \varphi^{2}\left(g^{-1}\right)=\varphi^{1}\left(g^{-1}\right) \circ h^{\diamond},
$$

completing the proof.

\section{Appendix B: The proof of Theorem 2.2}

We assume that $\varphi \in \mathcal{R}_{G}$ is type 1 and minimal, and will show that $\varphi$ is proximal, the other implication being obvious. Call a closed interval $I \subset S^{1} \varphi$ contactible if $\inf _{g \in G}|\varphi(g) I|=0$. First of all we have the following easy fact.

(1) For any $g \in G$ and any closed interval $I, I$ is $\varphi$-contractible if and only if $\varphi(g) I$ is $\varphi$-contractible.

Next let us show:

(2) There is $\delta>0$ such that if $|I|<\delta$, then $I$ is $\varphi$-contractible.

Proof. Since $\varphi$ is not type 0 , there is a nontrivial homeomorphism $\varphi(g)$ which admits a fixed point. This shows that there is a $\varphi$-contractible interval $J$. Since $\varphi$ is minimal, the family

$$
\mathcal{J}=\{\varphi(g) \operatorname{Int} J \mid g \in G\}
$$

must cover $S^{1}$. Now the Lebesgue number $\delta$ of the open covering $\mathcal{J}$ works.

Define a map $\widetilde{U}: \mathbb{R} \rightarrow \mathbb{R}$ by

$$
\widetilde{U}(\widetilde{x})=\sup \{\widetilde{y} \in(\widetilde{x}, \infty) \mid \pi([\widetilde{x}, \widetilde{y}]) \text { is } \varphi \text {-contractible }\} .
$$

We have the following easy properties.

(3) $\widetilde{x}+\delta \leq \widetilde{U}(\widetilde{x}) \leq \widetilde{x}+1$.

(4) $\widetilde{U}$ is monotone nondecreasing.

Also (1) implies the following.

(5) For any $g \in G$ and a lift $\widetilde{\varphi(g)}$ of $\varphi(g)$ to $\mathbb{R}$,

$$
\widetilde{\varphi(g)} \circ \widetilde{U}=\widetilde{U} \circ \widetilde{\varphi(g)} .
$$

Especially, $\widetilde{U} \circ T=T \circ \widetilde{U}$. 
(6) The map $\widetilde{U}$ is injective.

Proof. Assume on the contrary that there is $\widetilde{y} \in \mathbb{R}$ such that $\mathrm{Cl}\left(\widetilde{U}^{-1}(\widetilde{y})\right)=$ $\left[\widetilde{x}_{0}, \widetilde{x}_{1}\right]$ is an interval. By the minimality of $\varphi$, there is a lift $\widetilde{\varphi(g)}$ such that $\widetilde{\varphi(g)}\left(\widetilde{x}_{1}\right) \in\left(\widetilde{x}_{0}, \widetilde{x}_{1}\right)$. Then there is $\widetilde{x}_{2} \in\left(\widetilde{x}_{0}, \widetilde{x}_{1}\right)$ such that $\widetilde{\varphi(g)}\left(\widetilde{x}_{2}\right) \in\left(\widetilde{x}_{0}, \widetilde{x}_{1}\right)$ and $\widetilde{\varphi(g)}^{-1}\left(\widetilde{x}_{2}\right) \in\left(\widetilde{x}_{1}, \infty\right)$. Now

$$
\widetilde{\varphi(g)}(\widetilde{y})=\widetilde{\varphi(g)} \circ \widetilde{U}\left(\widetilde{x}_{2}\right)=\widetilde{U} \circ \widetilde{\varphi(g)}\left(\widetilde{x}_{2}\right)=\widetilde{y} .
$$

This shows

$$
\widetilde{U} \circ \widetilde{\varphi(g)}^{-1}\left(\widetilde{x}_{2}\right)=\widetilde{\varphi(g)}^{-1} \circ \widetilde{U}\left(\widetilde{x}_{2}\right)=\widetilde{\varphi(g)}^{-1}(\widetilde{y})=\widetilde{y} .
$$

This contradicts with the fact that $\widetilde{\varphi(g)}^{-1}\left(\widetilde{x}_{2}\right) \notin\left[\widetilde{x}_{0}, \widetilde{x}_{1}\right]=\operatorname{Cl}\left(\widetilde{U}^{-1}(\widetilde{y})\right)$.

(7) $\widetilde{U}$ is bijective.

Proof. Define $\widetilde{V}: \mathbb{R} \rightarrow \mathbb{R}$ by

$$
\widetilde{V}(\widetilde{x})=\inf \{\widetilde{y} \in(-\infty, \widetilde{x}) \mid \pi([\widetilde{y}, \widetilde{x}]) \text { is } \varphi \text {-contractible }\} .
$$

For any point $\widetilde{x} \in \mathbb{R}$, and any point $\widetilde{x}_{1}$ in $(\widetilde{x}, \widetilde{U}(\widetilde{x})),(6)$ implies that $\widetilde{U}(\widetilde{x})<\widetilde{U}\left(\widetilde{x}_{1}\right)$. This shows that the interval $\pi\left(\left[\widetilde{x}_{1}, \widetilde{U}(\widetilde{x})\right]\right)$ is $\varphi$-contractible. Since $\widetilde{x}_{1}$ is an arbitrary point of $(\widetilde{x}, \widetilde{U}(\widetilde{x}))$, this shows that $\widetilde{V}(\widetilde{U}(\widetilde{x})) \leq \widetilde{x}$. Again by (6), we have in fact

$$
\widetilde{V}(\widetilde{U}(\widetilde{x}))=\widetilde{x} .
$$

The same argument shows that $\widetilde{U} \circ \widetilde{V}=\mathrm{Id}$.

By (4) and (7), $\widetilde{U}$ is a homeomorphism. By (5), there is $U \in \mathcal{H}$ such that $\pi \circ \widetilde{U}=U \circ \pi$. Also by (5), $U$ commutes with any element of $\varphi(G)$. Finally let us show:

(8) There is $k \in \mathbb{N}$ such that $U^{k}=\mathrm{Id}$.

Proof. If $\operatorname{Fix}\left(U^{k}\right)$ is nonempty for some $k \in \mathbb{N}$, then $\operatorname{Fix}\left(U^{k}\right)$ must be invariant by $\varphi(G)$, since $U^{k}$ commutes with any element of $\varphi(G)$. That is, $\operatorname{Fix}\left(U^{k}\right)=S^{1}$, showing (8). If not, the rotation number of $U$ must be irrational, and there is a unique minimal set $X$ of $U$. Since $X$ is unique and since $U$ commutes with any element of $\varphi(G), X$ must be left invariant by any element of $\varphi(G)$. Since $\varphi$ is minimal, this implies $X=S^{1}$. That is, $U$ is topologically conjugate to an irrational rotation. But then $\varphi(G)$ must be abelian, and $\varphi$ must be of type 0. A contradiction.

To conclude, since $\varphi$ is assumed to be of type 1 , we have $k=1$. But by (3), this implies $\widetilde{U}=T$. That is, $\varphi$ is proximal.

\section{References}

[1] J. Bowden, Contact structures, deformations and taut foliations, Arxiv: 1304.3833.

[2] D. Calegari and A. Walker, Ziggurats and rotation numbers, J. Modern Dynamics 5(2011), 711-746.

[3] É. Ghys, Groups acting on the circle, l'Ens. Math. 47(2001), 329-407.

[4] K. Mann, Components of surface group representations, Arxiv: 1309.2905.

[5] B. Maskit, On Klein's combination theorem, Trans. A. M. S. 120(1965), 499-509.

[6] S. Matsumoto, Numerical invarinats for semi-conjugacy of homeomorphisms of the circle, Proc. A. M. S. 96(1986), 163-168.

[7] S. Matsumoto, Some remarks on foliated $S^{1}$ bundles, Invent. Math. 90(1987), 343-358.

[8] J. Milnor, On the existence of a connection with curvature zero, Comm. Math. Helv. 32(1958), 215-223. 
[9] J. Wood, Bundles with totally disconnected structure group, Comm. Math. Helv. 51(1971), 183-199.

Department of Mathematics, College of Science and Technology, Nihon UniverSity, 1-8-14 Kanda, Surugadai, Chiyoda-ku, Tokyo, 101-8308 Japan

E-mail address: matsumo@math.cst.nihon-u.ac.jp 